Research Article

\title{
Novel Technique for Group Decision-Making under Fuzzy Parameterized $q$-Rung Orthopair Fuzzy Soft Expert Framework
}

\author{
Ghous Ali $\mathbb{i D}^{1}$ and Musavarah Sarwar $\mathbb{D}^{2}$ \\ ${ }^{1}$ Department of Mathematics, Division of Science and Technology, University of Education, Lahore, Pakistan \\ ${ }^{2}$ Department of Mathematics, Government College Women University, Sialkot, Pakistan \\ Correspondence should be addressed to Musavarah Sarwar; musavarah656@gmail.com
}

Received 8 August 2021; Revised 10 September 2021; Accepted 30 September 2021; Published 27 October 2021

Academic Editor: Yong Aaron Tan

Copyright (C) 2021 Ghous Ali and Musavarah Sarwar. This is an open access article distributed under the Creative Commons Attribution License, which permits unrestricted use, distribution, and reproduction in any medium, provided the original work is properly cited.

\begin{abstract}
The q-rung orthopair fuzzy sets and their hybrid models are capable of dealing with uncertain situations very effectively than the theories of intuitionistic and Pythagorean fuzzy sets and thus have numerous decision-making applications in daily life, while the fuzzy parameterized soft set theory has its impact on different decision-making scenarios. Motivated by these facts, in this research article, these theories are combined to form a new structure named fuzzy parameterized $q$-rung orthopair fuzzy soft expert sets (FP $q$ ROFSESs) for dealing with more generalized information. The developed model is an efficient extension of fuzzy parameterized intuitionistic fuzzy soft expert sets. Some of its basic notions, including subset, complement, OR operation, AND operation, intersection, and union are studied and illustrated via examples. Moreover, to show the applicability and efficiency of the developed model, two real-life applications are solved under the $\mathrm{FP}^{q} \mathrm{ROFSES}$ approach, which is supported by an algorithm, the first application is about selecting an appropriate site for a cafe outlet, and the second application is about selecting the Best News Channel for an award. At last, a comparison of the initiated model with some existing approaches is presented to verify its advantages over them.
\end{abstract}

\section{Introduction}

Nowadays, a lot of researchers and scientists across the globe keep on working to find the solutions to complexities and situations unsolvable by traditional mathematical tools; for example, crisp set theory is not capable of dealing with different real-world problems concerning uncertainties in various areas, including engineering, medicine, and artificial intelligence. A solution to these problems emerged as the notion of fuzzy sets initiated by Zadeh [1] in 1965. Instead of normally declaring a belongingness degree (i.e., 1) or a nonbelongingness degree (i.e., 0) of an element in the classical set theory, fuzzy sets allow partial belongingness degrees from the interval $[0,1]$ to be assigned to each element, thus claiming its vague boundary scenario by extending crisp set theory. This powerful concept fills the gaps in the previous traditional concepts allowing modeling of and solution to many vague situations. Inspection of the last few decades leads us to an important fact that the fuzzy set model urged many scientists and experts to use and extend this model for solving numerous uncertain problems.

In a fuzzy set, the nonbelongingness degree is dependent on the belongingness degree and calculated as " 1 minus belongingness degree." However, there come situations where belongingness and nonbelongingness degrees may vary from this criterion. To tackle this difficulty, Atanassov [2] proposed intuitionistic fuzzy sets (IFSs) as an extension to fuzzy sets by providing two degrees, i.e., the belongingness degree $\alpha_{I}$ and nonbelongingness degree $\beta_{I}$ for an element with the constraint that $0 \leq \alpha_{I}+\beta_{I} \leq 1$. Thus, it allows dealing better with the uncertainties, e.g., a situation where $\alpha_{I}=0.3$ and $\beta_{I}=0.6$. But this model fails to deal with situations where belongingness and nonbelongingness degrees sum up above unity. For this, Yager [3] initiated the concept of Pythagorean fuzzy sets (PFSs) as a generalization of IFSs, allowing higher applicability in two-dimensional 
uncertainties. The belongingness degree $\alpha_{P}$ and nonbelongingness degree $\beta_{P}$ are now conditioned with the constraint $0 \leq\left(\alpha_{P}\right)^{2}+\left(\beta_{P}\right)^{2} \leq 1$. Due to their higher ability to deal with uncertainties than IFSs, they have been utilized widely in many decision-making situations. Later on, Senapati and Yager [4] observed that in a particular situation where a belongingness degree of 0.65 and a nonbelongingness degree of 0.85 are assigned to an element, then $(0.65)^{2}+(0.85)^{2}=1.145 \nless 1$. Hence, PFSs fail to deal with it. To deal with such situations, Senapati and Yager [4] extended the PFSs to Fermatean fuzzy sets (FFSs) with the condition $0 \leq\left(\alpha_{F}\right)^{3}+\left(\beta_{F}\right)^{3} \leq 1$. Thus, this increased order of uncertainties allows handling of the problems as discussed above since $(0.65)^{3}+(0.85)^{3}=0.888 \leq 1$. Afterward, Yager's [5] contribution came in the form of $q$-rung orthopair fuzzy sets ( $q$-ROFSs) or generalized orthopair fuzzy sets with the characteristic of the sum of the $q$ th power of belongingness and nonbelongingness values of elements not being more than one. The $q$-ROFSs are generally reduced to IFSs, PFSs, and FFSs for $q=1,2$ and $q=3$, respectively (see Figure 1). Later, Shaheen et al. [6] briefly investigated the reasons behind the construction of $q$-ROFSs. A number of decision-making problems have been solved by using the $q$-ROFS model [7-11].

All the models mentioned above have a common limitation in that they fail to deal with situations considering multiple parameters. To deal with this issue, Molodtsov [12] initiated the concept of soft set theory that provides parameterization tools for handling uncertainties. Actually, soft sets are modeled as parameterized families of sets, thus giving a parameterized methodology for multiattribute decision-making problems. Maji et al. [13] discussed some properties and operations of soft sets. The concepts of the soft set model were naturally extended and combined with other models by many experts to deal with uncertain situations. Some important models are fuzzy soft sets (FSSs) [14] and intuitionistic FSSs [15]. These models fail to deal with some practical situations. To overcome this difficulty, Hamid et al. [16] generalized intuitionistic FSSs and presented the idea of a novel hybrid model called $q$-ROF soft sets ( $q$-ROFSSs). There is a deficiency in this model; that is, it cannot deal with bipolar information. In order to handle this issue, very recently, Ali et al. [17] presented a novel hybrid multi-criteria decision-making (MCDM) model, namely, $q$-ROF bipolar soft sets as a generalization of $q$-ROFSSs. In addition, Alkan and Kahraman [18] proposed a q-rung orthopair fuzzy TOPSIS method for the evaluation of government strategies against the COVID-19 pandemic.

In the soft set model, elements are categorized with respect to the parameters. However, it is seen that in most cases, some parameters have more preferences over others, and thus, higher degrees of less preferable parameter families may affect the decisions. For this, fuzzy parameterized soft sets were introduced by Aman and Enginoglu [19], where fuzzy memberships are assigned to the parameters which better demonstrate the weightage or the relative preferences of the parameters. In addition, the same authors extended it to fuzzy parameterized fuzzy soft sets (FPFSSs) [20]. This MCDM model fails to deal with data in an intuitionistic

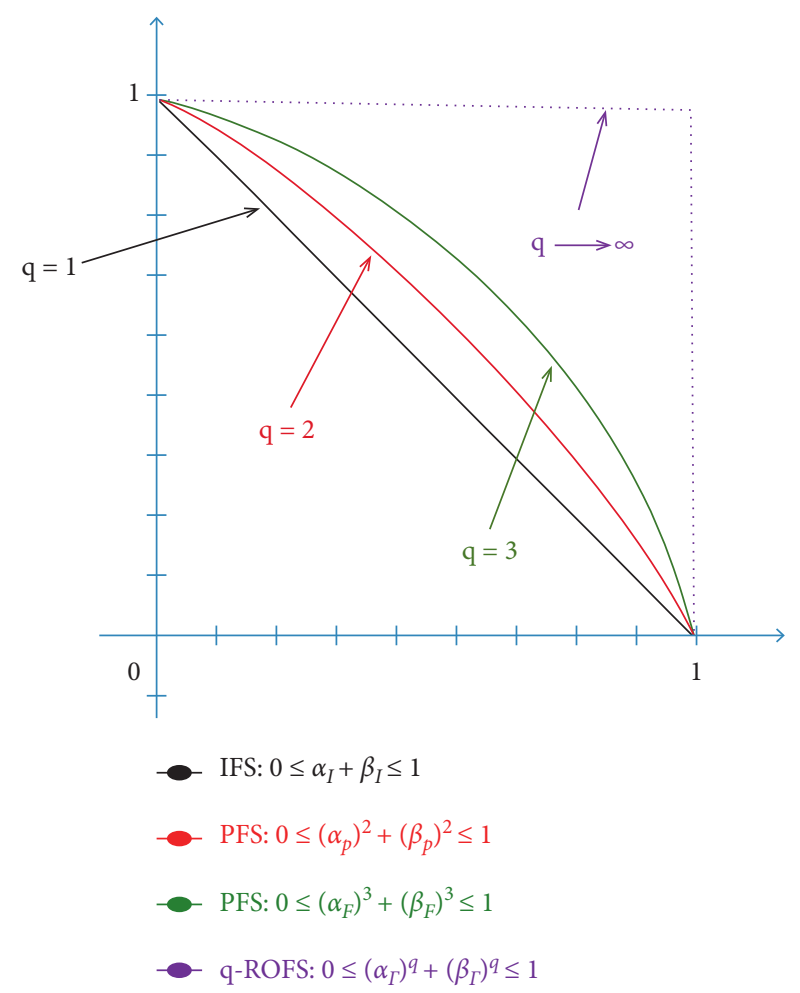

FIGURE 1: Comparison between the spaces of IFSs, PFSs, FFSs, and $q$-ROFSs.

fuzzy environment. That is why more improvements and extensions to this fruitful concept include intuitionistic fuzzy parameterized soft sets (IFPSSs) [21], intuitionistic fuzzy parameterized FSSs (IFPFSSs) [22], and intuitionistic fuzzy parameterized intuitionistic FSSs (IFPIFSSs) [23, 24]. All the mentioned above fuzzy parameterized soft models are not suitable in the case of interval-valued representation of data and information. To overcome this difficulty, recently, Aydin and Enginolu [25] presented a more generalized model called interval-valued intuitionistic fuzzy parameterized interval-valued intuitionistic FSSs (IVIFP-IVIFSSs) and solved a decision-making application.

Since the models and their hybridization discussed above have vital importance in handling uncertainties, one common restriction is that they deal with a single expert. However, many situations require multiple experts opinions or group decision-making. For instance, when dealing with the selection of an admin manager in a company, the committee of two or more people takes its judgments as scores on questionnaires and finally declares who will be the most suitable person. To deal with such scenarios, Alkhazaleh and Salleh [26] introduced soft expert sets (SESs), which are capable of integrating the opinions of all experts in one place and hence are extremely efficient in multiattribute group decision-making (MAGDM) situations. Later, the same authors discussed the fuzziness of the SES model [27]. Due to group decision-making modeling capabilities of SESs, many hybrid models have been proposed till now as SESs are their major component, fuzzy $N$-SESs [28], fuzzy parameterized intuitionistic fuzzy SESs [29], fuzzy bipolar 
soft expert sets [30], analysis of chat conversations of pedophiles based on bipolar fuzzy soft sets [31], risk assessment in automatic robots using rough ELECTRE-II approach [32], and decision support systems based on rough DTOPSIS method [33]. Akram et al. [34] developed a new hybrid model called $m$-polar fuzzy SESs, which discusses the multipolarity of fuzzy SESs and solved some group decisionmaking problems. For more useful terms, the reader is referred to [35-44].

1.1. Motivation and Contribution. The motives of the current study are summarized as follows:

(1) There is a considerably wider applicability scope of $q$-ROFSs as compared to IFSs, PFSs, and FFSs in dealing with 2-dimensional uncertainties. To better understand this argument, consider a pair containing belongingness and nonbelongingness degrees as $(0.80,0.95)$; then, it is clear that $0.80+0.95 \nless 1,(0.80)^{2}+(0.95)^{2} \quad \nless 1,(0.80)^{3}+$ $(0.95)^{3} \nless 1$. But $(0.80)^{q}+(0.95)^{q}<1$ for all $q \geq 6$.

(2) There are parameterization capabilities of fuzzy parameterized intuitionistic fuzzy SESs (FPIFSESs) for dealing with IFS information under multiple experts with weighted preferences.

(3) The inability of FPIFSESs to deal with $q$-ROF information indicates the need for an extension of this model, which also preserves the existing abilities of the FPIFSES model.

(4) The $q$-ROFS model was found to be more effective when extended to the range of parameterizations and used in different domains, that is, $q$-ROFSSs but the main drawback of this model is that it has no ability to tackle a situation where different weights are assigned to different parameters in $q$-ROF information. Actually, the fuzzy parameterized versions of $q$-ROFSSs and $q$-ROFSESs are unattended to date.

Motivated by the above analysis, in this research article, the concept of FPIFSESs or $q$-ROFSSs is extended to $\mathrm{FP}^{q}$ ROFSESs, thus allowing more uncertainties to be handled easily as the order of uncertainty is increased from 1 to $q$ th power of belongingness and nonbelongingness degrees.

This research contributes the following:

(1) A new and powerful extension of the FPIFSES model is provided, namely, $\mathrm{FP}^{q}$ ROFSES, which allows dealing with $q$-ROFS information efficiently

(2) Basic operations, including subset, complement, OR operation, AND operation, intersection, and union of the newly developed model, are provided and supported with examples

(3) Two real-life MAGDM problems, including the best site selection for a new cafe outlet and the selection of Best News Channel, are solved using a developed algorithm based on $\mathrm{FP}^{q}$ ROFSESs
(4) A comparison of the developed group decisionmaking method under $\mathrm{FP}^{q}$ ROFSESs with few existing approaches is also given

1.2. Framework of the Paper. Section 1 includes the introduction, related works, motivation, and contribution of the research article. Section 2 recalls some basic definitions and then introduces the main concept of $\mathrm{FP}^{q}$ ROFSESs along with certain essential notions and basic operations for the $\mathrm{FP}^{q}$ ROFSESs. Section 3 presents two real-life applications of the $\mathrm{FP}^{q}$ ROFSESs with an algorithmic approach. Section 4 discusses a comparison of the developed method under $\mathrm{FP}^{q}$ ROFSESs with few existing approaches. At last, Section 5 gives the concluding remarks and some future directions.

\section{Fuzzy Parameterized $q$-Rung Orthopair Fuzzy Soft Expert Sets}

This section introduces the $\mathrm{FP}^{q}$ ROFSES model, its basic operations, and its properties. But before that, we need to review some essential definitions useful for its construction and further study throughout the article.

Definition 1 (see [5]). Let $\mathscr{Y}$ be a universal set. A pair $\mathscr{G}=$ $\left(\alpha_{\Gamma}, \beta_{\Gamma}\right)$ is called a $q$-rung orthopair fuzzy set or $q$-ROFS over $y$ where $\alpha_{\Gamma}$ is a membership function given by $\alpha_{\Gamma}: \mathscr{Y} \longrightarrow[0,1]$ and $\beta_{\Gamma}$ is a nonmembership function given by $\beta_{\Gamma}: \mathcal{Y} \longrightarrow[0,1]$ with $0 \leq\left(\alpha_{\Gamma}(\mathfrak{y})\right)^{q}+\left(\beta_{\Gamma}(\mathfrak{y})\right)^{q} \leq 1$ where $q \geq 1, \alpha_{\Gamma}(\mathfrak{y}), \beta_{\Gamma}(\mathfrak{y}) \in[0,1]$ for all $\mathfrak{y} \in \mathscr{Y}$. In set form, a $q$ ROFS on $y$ is given as

$$
\mathscr{G}=\left\{\left\langle\mathfrak{y},\left(\alpha_{\Gamma}(\mathfrak{y}), \beta_{\Gamma}(\mathfrak{y})\right)\right\rangle \mid \mathfrak{y} \in \mathscr{Y}\right\},
$$

where $\alpha_{\Gamma}(\mathfrak{y}), \beta_{\Gamma}(\mathfrak{y}) \in[0,1]$ denotes the belongingness and nonbelongingness values, respectively, and satisfies $0 \leq\left(\alpha_{\Gamma}(\mathfrak{y})\right)^{q}+\left(\beta_{\Gamma}(\mathfrak{y})\right)^{q} \leq 1$. Moreover, $\left(\alpha_{\Gamma}(\mathfrak{y}), \beta_{\Gamma}(\mathfrak{y})\right)$ is known as a $q$-rung orthopair fuzzy number $(q$-ROFN) and denoted by $\mathbb{Q}=\left(\alpha_{\Gamma}(\mathfrak{y}), \beta_{\Gamma}(\mathfrak{y})\right)$. The degree of hesitance for $q$-ROFN $\mathbb{Q}=\left(\alpha_{\Gamma}(\mathfrak{y}), \beta_{\Gamma}(\mathfrak{y})\right)$ is defined by

$$
\pi_{\mathscr{Q}}=\sqrt[q]{1-\left(\left(\alpha_{\Gamma}(\mathfrak{y})\right)^{q}+\left(\beta_{\Gamma}(\mathfrak{y})\right)^{q}\right)} .
$$

Definition 2 (see [16]). Let $\mathcal{Y}$ be a universe of discourse and let $\mathcal{S}$ be a set of parameters. Let $\mathscr{A} \subseteq \mathcal{S}$ and $\mathfrak{Q}^{\mathcal{Y}}$ be a collection of all $q$-ROF subsets of $\mathcal{S}$. A pair $(\Gamma, \mathscr{A})$ is said to be a $q$-rung orthopair fuzzy soft set ( $q$-ROFSS) on $\mathcal{Y}$, if $\Gamma$ is the function defined by $\Gamma: \mathscr{A} \longrightarrow \mathfrak{Q}^{\mathscr{Y}}$.

For $\mathfrak{y} \in \mathscr{Y}$ and $\mathfrak{g} \in \mathscr{A}$, a $q$-ROFS $\Gamma(\mathfrak{g})$ is given as

$$
\Gamma(\mathfrak{g})=\left\{\left\langle\mathfrak{y},\left(\alpha_{\Gamma}(\mathfrak{g})(\mathfrak{y}), \beta_{\Gamma}(\mathfrak{g})(\mathfrak{y})\right)\right\rangle \mid \mathfrak{y} \in \mathscr{Y}\right\},
$$

such that the belongingness degree " $\alpha_{\Gamma}$ " and nonbelongingness degree " $\beta_{\Gamma}$ " are conditioned with the constraint $0 \leq\left(\alpha_{\Gamma}(\mathfrak{g})(\mathfrak{y})\right)^{q}+\left(\beta_{\Gamma}(\mathfrak{g})(\mathfrak{y})\right)^{q} \leq 1$, for all $q \in(0, \infty)$.

Definition 3 (see [29]). Let $\mathcal{Y}$ be a universe, let $\mathcal{S}$ be a set of parameters, let $\mathscr{E}$ be a set of experts, and let $\mathcal{O}=\{0=$ disagree, $1=$ agree $\}$ be their set of opinions. Let $\mathscr{D}$ be the fuzzy subset of $\mathcal{S}$, and 
$\mathscr{Z} \subseteq \mathscr{D} \times \mathscr{E} \times \mathscr{O}=\{(\mathfrak{d}, \mathfrak{e}, \mathfrak{v}) \mid \mathfrak{d} \in \mathscr{D}, \mathfrak{e} \in \mathscr{E}, \mathfrak{v} \in \mathscr{O}\}$. Consider a mapping $\mathfrak{I}: \mathscr{Z} \longrightarrow \mathrm{IF}^{\mathscr{Y}}$, where $\mathrm{IF}^{\mathscr{Y}}$ is the collection of all IFSs on $\mathscr{Y}$. A pair $(\mathfrak{I}, \mathscr{Z})_{\mathscr{D}}$ is called a fuzzy parameterized intuitionistic fuzzy soft expert set or FPIFSES, where

$$
(\mathfrak{I}, \mathscr{Z})_{\mathscr{D}}=\{(\mathfrak{z}, \mathfrak{I}(\mathfrak{z})): \mathfrak{z} \in \mathscr{Z}\},
$$

such that $\mathfrak{I}(\mathfrak{z})=\left\{\left(\mathfrak{y} /\left(\alpha_{\mathfrak{J}}(\mathfrak{z})(\mathfrak{y}), \beta_{\mathfrak{I}}(\mathfrak{z})(\mathfrak{y})\right)\right) \mid \mathfrak{y} \in \mathscr{Y}\right\}$ with $0 \leq\left(\alpha_{\mathfrak{J}}(\mathfrak{z})(\mathfrak{y})\right)^{q}+\left(\beta_{\mathfrak{J}}(\mathfrak{z})(\mathfrak{y})\right)^{q} \leq 1$.

We are now ready to construct the notion of novel $\mathrm{FP}^{q}$ ROFSESs, which is given as follows.

Definition 4. Let $\mathcal{Y}$ be a universe, let $\delta$ be a set of parameters, let $\mathscr{E}$ be a set of experts, and let $\mathcal{O}=\{0=$ disagree, $1=$ agree $\}$ be their set of opinions. Let $\mathscr{D}$ be the fuzzy subset of $\delta$, and $\mathscr{Z} \subseteq \mathscr{D} \times \mathscr{E} \times \mathscr{O}=\{(\mathfrak{D}, \mathfrak{e}, \mathfrak{v}) \mid \mathfrak{d} \in \mathscr{D}, \mathfrak{e} \in \mathscr{E}, \mathfrak{v} \in \mathscr{O}\}$. Consider a mapping $\mathfrak{f}: \mathscr{Z} \longrightarrow \mathfrak{Q}^{\mathscr{Y}}$, where $\mathfrak{Q}^{\mathscr{Y}}$ is the collection of all $q$-ROFSs on $\mathscr{Y}$. A pair $(\mathfrak{f}, \mathscr{Z})_{\mathscr{D}}$ is said to be a fuzzy parameterized $q$-rung orthopair fuzzy soft expert set or $\mathrm{FP}^{q}$ ROFSES, where

$$
(\mathfrak{f}, \mathscr{Z})_{\mathscr{D}}=\{(\mathfrak{z}, \mathfrak{f}(\mathfrak{z})): \mathfrak{z} \in \mathscr{Z}\},
$$

such that $\mathfrak{f}(\mathfrak{z})=\left\{\left(\mathfrak{y} /\left(\alpha_{\mathfrak{f}}(\mathfrak{z})(\mathfrak{y}), \beta_{\mathfrak{f}}(\mathfrak{z})(\mathfrak{y})\right)\right) \mid \mathfrak{y} \in \mathscr{Y}\right\}$ with $0 \leq\left(\alpha_{\mathfrak{f}}(\mathfrak{z})(\mathfrak{y})\right)^{q}+\left(\beta_{\mathfrak{f}}(\mathfrak{z})(\mathfrak{y})\right)^{q} \leq 1$, for all $q \in(0, \infty)$.

This main concept is now explained by an example given as follows.

Example 1. Consider a person is interested in buying a house, but before spending on the house, he needs to be sure that the house meets all his needs. In such a condition, he decides to consider the opinions of experts in selecting a reasonable house. Consider there are four houses available in the desired area for purchase, constituting the universal set $\mathscr{Y}=\left\{\mathfrak{y}_{1}, \mathfrak{y}_{2}, \mathfrak{y}_{3}, \mathfrak{y}_{4}\right\}$. The person contacts two experts as in the set $\mathscr{E}=\left\{\mathfrak{e}_{1}, \mathfrak{e}_{2}\right\}$, for helping him in making the decision. These experts consider the following parameters $\mathcal{S}=\left\{\mathfrak{I}_{1}, \mathfrak{g}_{2}, \mathfrak{g}_{3}, \mathfrak{I}_{4}\right\}, \quad$ where $\mathfrak{g}_{1}=$ cheap, $\quad \mathfrak{g}_{2}=$ beautiful, $\mathfrak{g}_{3}=$ expensive, and $\mathfrak{g}_{4}=$ materal, for the selection of the house. Let $\mathscr{D}=\left\{\left(0.6 / \mathfrak{g}_{1}\right),\left(0.7 / \mathfrak{g}_{2}\right),\left(0.9 / \mathfrak{g}_{3}\right),\left(0.2 / \mathfrak{g}_{4}\right)\right\}$ represents certain weights for the parameters according to the buyer's requirements and $q=4$. Then, the $\mathrm{FP}^{4}$ ROFSES $(\mathfrak{f}, \mathscr{Z})_{\mathscr{D}}$ representing the opinions of experts about the houses regarding parameters is described as follows:

$$
\begin{aligned}
(\mathfrak{f}, \mathfrak{Z})_{\mathscr{D}}= & \left\langle\left(\frac{0.6}{\mathfrak{g}_{1}}, \mathfrak{e}_{1}, 1\right),\left\{\frac{\mathfrak{y}_{1}}{(0.30,0.60)}, \frac{\mathfrak{y}_{2}}{(0.80,0.70)}, \frac{\mathfrak{y}_{3}}{(0.20,0.10)}, \frac{\mathfrak{y}_{4}}{(0.50,0.20)}\right\}\right\rangle, \\
& \left\langle\left(\frac{0.7}{\mathfrak{g}_{2}}, \mathfrak{e}_{1}, 1\right),\left\{\frac{\mathfrak{y}_{1}}{(0.70,0.90)}, \frac{\mathfrak{y}_{2}}{(0.60,0.10)}, \frac{\mathfrak{y}_{3}}{(0.30,0.20)}, \frac{\mathfrak{y}_{4}}{(0.10,0.40)}\right\}\right\rangle, \\
& \left\langle\left(\frac{0.9}{\mathfrak{g}_{3}}, \mathfrak{e}_{1}, 1\right),\left\{\frac{\mathfrak{y}_{1}}{(0.10,0.50)}, \frac{\mathfrak{y}_{2}}{(0.80,0.10)}, \frac{\mathfrak{y}_{3}}{(0.80,0.20)}, \frac{\mathfrak{y}_{4}}{(0.30,0.80)}\right\}\right\rangle, \\
& \left\langle\left(\frac{0.2}{\mathfrak{g}_{4}}, \mathfrak{e}_{1}, 1\right),\left\{\frac{\mathfrak{y}_{1}}{(0.10,0.20)}, \frac{\mathfrak{y}_{2}}{(0.70,0.30)}, \frac{\mathfrak{y}_{3}}{(0.70,0.40)}, \frac{\mathfrak{y}_{4}}{(0.90,0.60)}\right\}\right\rangle, \\
& \left\langle\left(\frac{0.6}{\mathfrak{g}_{1}}, \mathfrak{e}_{2}, 1\right),\left\{\frac{\mathfrak{y}_{1}}{(0.80,0.60)}, \frac{\mathfrak{y}_{2}}{(0.80,0.70)}, \frac{\mathfrak{y}_{3}}{(0.80,0.50)}, \frac{\mathfrak{y}_{4}}{(0.80,0.60)}\right\}\right\rangle, \\
& \left\langle\left(\frac{0.7}{\mathfrak{g}_{2}}, \mathfrak{e}_{2}, 1\right),\left\{\frac{\mathfrak{y}_{1}}{(0.90,0.16)}, \frac{\mathfrak{y}_{2}}{(0.50,0.15)}, \frac{\mathfrak{y}_{3}}{(0.40,0.14)}, \frac{\mathfrak{y}_{4}}{(0.30,0.14)}\right\}\right\rangle, \\
& \left\langle\left(\frac{0.9}{\mathfrak{g}_{3}}, \mathfrak{e}_{2}, 1\right),\left\{\frac{\mathfrak{y}_{1}}{(0.66,0.36)}, \frac{\mathfrak{y}_{2}}{(0.44,0.72)}, \frac{\mathfrak{y}_{3}}{(0.48,0.52)}, \frac{\mathfrak{y}_{4}}{(0.36,0.44)}\right\}\right\rangle, \\
& \left\langle\left(\frac{0.2}{\mathfrak{g}_{4}}, \mathfrak{e}_{2}, 1\right),\left\{\frac{\mathfrak{y}_{1}}{(0.87,0.45)}, \frac{\mathfrak{y}_{2}}{(0.45,0.56)}, \frac{\mathfrak{y}_{3}}{(0.79,0.36)}, \frac{\mathfrak{y}_{4}}{(0.62,0.42)}\right\}\right\rangle, \\
& \left\langle\left(\frac{0.6}{\mathfrak{g}_{1}}, \mathfrak{e}_{1}, 0\right),\left\{\frac{\mathfrak{y}_{1}}{(0.70,0.40)}, \frac{\mathfrak{y}_{2}}{(0.60,0.30)}, \frac{\mathfrak{y}_{3}}{(0.70,0.40)}, \frac{\mathfrak{y}_{4}}{(0.80,0.20)}\right\}\right\rangle, \\
& \left\langle\left(\frac{0.7}{\mathfrak{g}_{2}}, \mathfrak{e}_{1}, 0\right),\left\{\frac{\mathfrak{y}_{1}}{(0.70,0.10)}, \frac{\mathfrak{y}_{2}}{(0.80,0.60)}, \frac{\mathfrak{y}_{3}}{(0.40,0.14)}, \frac{\mathfrak{y}_{4}}{(0.56,0.65)}\right\}\right\rangle,
\end{aligned}
$$




$$
\begin{aligned}
& \left\langle\left(\frac{0.9}{\mathfrak{g}_{3}}, \mathfrak{e}_{1}, 0\right),\left\{\frac{\mathfrak{y}_{1}}{(0.80,0.70)}, \frac{\mathfrak{y}_{2}}{(0.90,0.60)}, \frac{\mathfrak{y}_{3}}{(0.80,0.20)}, \frac{\mathfrak{y}_{4}}{(0.80,0.30)}\right\}\right\rangle, \\
& \left\langle\left(\frac{0.2}{\mathfrak{G}_{4}}, \mathfrak{e}_{1}, 0\right),\left\{\frac{\mathfrak{y}_{1}}{(0.50,0.15)}, \frac{\mathfrak{y}_{2}}{(0.77,0.63)}, \frac{\mathfrak{y}_{3}}{(0.56,0.56)}, \frac{\mathfrak{y}_{4}}{(0.27,0.31)}\right\}\right\rangle, \\
& \left\langle\left(\frac{0.6}{\mathfrak{g}_{1}}, \mathfrak{e}_{2}, 0\right),\left\{\frac{\mathfrak{y}_{1}}{(0.90,0.60)}, \frac{\mathfrak{y}_{2}}{(0.62,0.42)}, \frac{\mathfrak{y}_{3}}{(0.71,0.81)}, \frac{\mathfrak{y}_{4}}{(0.72,0.44)}\right\}\right\rangle, \\
& \left\langle\left(\frac{0.7}{\mathfrak{g}_{2}}, \mathfrak{e}_{2}, 0\right),\left\{\frac{\mathfrak{y}_{1}}{(0.40,0.14)}, \frac{\mathfrak{y}_{2}}{(0.53,0.61)}, \frac{\mathfrak{y}_{3}}{(0.71,0.81)}, \frac{\mathfrak{y}_{4}}{(0.90,0.16)}\right\}\right\rangle, \\
& \left\langle\left(\frac{0.9}{\mathfrak{g}_{3}}, \mathfrak{e}_{2}, 0\right),\left\{\frac{\mathfrak{y}_{1}}{(0.72,0.42)}, \frac{\mathfrak{y}_{2}}{(0.77,0.63)}, \frac{\mathfrak{y}_{3}}{(0.50,0.80)}, \frac{\mathfrak{y}_{4}}{(0.30,0.80)}\right\}\right\rangle, \\
& \left.\left\langle\left(\frac{0.2}{\mathfrak{g}_{4}}, \mathfrak{e}_{2}, 0\right),\left\{\frac{\mathfrak{y}_{1}}{(0.30,0.80)}, \frac{\mathfrak{y}_{2}}{(0.79,0.36)}, \frac{\mathfrak{y}_{3}}{(0.80,0.70)}, \frac{\mathfrak{y}_{4}}{(0.50,0.16)}\right\}\right\rangle\right\} .
\end{aligned}
$$

Now, we discuss some essential basic properties of $\mathrm{FP}^{q}$ ROFSESs together with illustrative numerical examples. We start with subset relation.

Definition 5. For any two $\operatorname{FP}^{q}$ ROFSESs $(\mathfrak{f}, \mathscr{Z})_{\mathscr{D}}$ and $(\mathfrak{g}, \mathscr{W})_{\mathscr{K}}$ over $\mathscr{Y}$, the $\operatorname{FP}^{q} \operatorname{ROFSES}(\mathfrak{f}, \mathscr{Z})_{\mathscr{D}}$ is referred to as the fuzzy parameterized $q$-ROF soft expert subset of $(\mathfrak{g}, \mathscr{W})_{\mathscr{K}}$ if

(1) $\mathscr{Z} \subseteq \mathscr{W}$

(2) For all $\mathfrak{z} \in \mathscr{Z}, f_{\mathscr{D}}(\mathfrak{z})$ is $q$-ROF subset of $g_{\mathscr{K}}(\mathfrak{z})$
This subset relation is shown as $(\mathfrak{f}, \mathscr{Z})_{\mathscr{D}} \subset(\mathfrak{g}, \mathscr{W})_{\mathscr{K}}$, whereas $(\mathfrak{g}, \mathscr{W})_{\mathscr{K}}$ is called a fuzzy parameterized $q$-ROF soft expert superset of $(\mathfrak{f}, \mathscr{Z})_{\mathscr{D}}$.

Definition 6. Let $(\mathfrak{f}, \mathscr{Z})_{\mathscr{D}}$ and $(\mathfrak{g}, \mathscr{W})_{\mathscr{K}}$ be $\mathrm{FP}^{q}$ ROFSESs over a universe $\mathscr{Y}$. Then, $(\mathfrak{f}, \mathscr{Z})_{\mathscr{D}}$ and $(\mathfrak{g}, \mathscr{W})_{\mathscr{K}}$ are called equal if $(\mathfrak{f}, \mathscr{Z})_{\mathscr{D}}$ is a fuzzy parameterized $q$-ROF soft expert subset of $(\mathfrak{g}, \mathscr{W})_{\mathscr{K}}$ and $(g, \mathscr{W})_{\mathscr{K}}$ is a fuzzy parameterized $q$-ROF soft expert subset of $(\mathfrak{f}, \mathscr{Z})_{\mathscr{D}}$.

Example 2. Considering Example 1, suppose that a second opinion of the experts is taken once again for buying the house as follows:

$$
\begin{aligned}
& \mathscr{Z}=\left\{\left(\frac{0.6}{\mathfrak{g}_{1}}, \mathfrak{e}_{1}, 1\right),\left(\frac{0.7}{\mathfrak{g}_{2}}, \mathfrak{e}_{1}, 0\right),\left(\frac{0.6}{\mathfrak{g}_{1}}, \mathfrak{e}_{2}, 1\right),\left(\frac{0.7}{\mathfrak{g}_{2}}, \mathfrak{e}_{2}, 1\right),\left(\frac{0.6}{\mathfrak{g}_{1}}, \mathfrak{e}_{3}, 0\right),\left(\frac{0.7}{\mathfrak{g}_{2}}, \mathfrak{e}_{3}, 1\right)\right\}, \\
& \mathscr{W}=\left\{\left(\frac{0.8}{\mathfrak{g}_{1}}, \mathfrak{e}_{1}, 1\right),\left(\frac{0.9}{\mathfrak{g}_{2}}, \mathfrak{e}_{1}, 0\right),\left(\frac{0.3}{\mathfrak{g}_{3}}, \mathfrak{e}_{1}, 1\right),\left(\frac{0.8}{\mathfrak{g}_{1}}, \mathfrak{e}_{2}, 1\right),\left(\frac{0.9}{\mathfrak{g}_{2}}, \mathfrak{e}_{2}, 1\right),\left(\frac{0.8}{\mathfrak{g}_{1}}, \mathfrak{e}_{3}, 0\right),\left(\frac{0.9}{\mathfrak{g}_{2}}, \mathfrak{e}_{3}, 1\right),\left(\frac{0.3}{\mathfrak{g}_{3}}, \mathfrak{e}_{3}, 1\right)\right\}
\end{aligned}
$$

Clearly, $\mathscr{Z} \subseteq \mathscr{W}$. Consider two $\mathrm{FP}^{4}$ ROFSESs $(\mathfrak{f}, \mathscr{Z})_{\mathscr{D}}$ and $(\mathfrak{g}, \mathscr{W})_{\mathscr{K}}$ to be defined as follows:

$$
\begin{aligned}
(\mathfrak{f}, \mathscr{Z})_{\mathscr{D}}= & \left\{\left\langle\left(\frac{0.6}{\mathfrak{S}_{1}}, \mathfrak{e}_{1}, 1\right),\left\{\frac{\mathfrak{y}_{1}}{(0.80,0.85)}, \frac{\mathfrak{y}_{2}}{(0.70,0.65)}, \frac{\mathfrak{y}_{3}}{(0.90,0.35)}, \frac{\mathfrak{y}_{4}}{(0.50,0.80)}\right\}\right\rangle,\right. \\
& \left\langle\left(\frac{0.7}{\mathfrak{g}_{2}}, \mathfrak{e}_{1}, 0\right),\left\{\frac{\mathfrak{y}_{1}}{(0.19,0.40)}, \frac{\mathfrak{y}_{2}}{(0.60,0.70)}, \frac{\mathfrak{y}_{3}}{(0.12,0.55)}, \frac{\mathfrak{y}_{4}}{(0.22,0.50)}\right\}\right\rangle, \\
& \left\langle\left(\frac{0.6}{\mathfrak{g}_{1}}, \mathfrak{e}_{2}, 1\right),\left\{\frac{\mathfrak{y}_{1}}{(0.23,0.47)}, \frac{\mathfrak{y}_{2}}{(0.25,0.80)}, \frac{\mathfrak{y}_{3}}{(0.12,0.59)}, \frac{\mathfrak{y}_{4}}{(0.23,0.85)}\right\}\right\rangle,
\end{aligned}
$$




$$
\begin{aligned}
& \left\langle\left(\frac{0.7}{\mathfrak{g}_{2}}, \mathfrak{e}_{2}, 1\right),\left\{\frac{\mathfrak{y}_{1}}{(0.43,0.40)}, \frac{\mathfrak{y}_{2}}{(0.44,0.79)}, \frac{\mathfrak{y}_{3}}{(0.48,0.50)}, \frac{\mathfrak{y}_{4}}{(0.50,0.60)}\right\}\right\rangle \\
& \left\langle\left(\frac{0.6}{\mathfrak{g}_{1}}, \mathfrak{e}_{3}, 0\right),\left\{\frac{\mathfrak{y}_{1}}{(0.35,0.70)}, \frac{\mathfrak{y}_{2}}{(0.37,0.80)}, \frac{\mathfrak{y}_{3}}{(0.38,0.77)}, \frac{\mathfrak{y}_{4}}{(0.45,0.30)}\right\}\right\rangle, \\
& \left.\left\langle\left(\frac{0.7}{\mathfrak{g}_{2}}, \mathfrak{e}_{3}, 1\right),\left\{\frac{\mathfrak{y}_{1}}{(0.54,0.80)}, \frac{\mathfrak{y}_{2}}{(0.57,0.93)}, \frac{\mathfrak{y}_{3}}{(0.61,0.40)}, \frac{\mathfrak{y}_{4}}{(0.65,0.85)}\right\}\right\rangle\right\} . \\
& (\mathfrak{g}, \mathscr{W})_{\mathscr{K}}=\left\{\left\langle\left(\frac{0.8}{\mathfrak{g}_{1}}, \mathfrak{e}_{1}, 1\right),\left\{\frac{\mathfrak{y}_{1}}{(0.90,0.60)}, \frac{\mathfrak{y}_{2}}{(0.80,0.60)}, \frac{\mathfrak{y}_{3}}{(0.91,0.32)}, \frac{\mathfrak{y}_{4}}{(0.60,0.70)}\right\}\right\rangle,\right. \\
& \left\langle\left(\frac{0.9}{\mathfrak{S}_{2}}, \mathfrak{e}_{1}, 0\right),\left\{\frac{\mathfrak{y}_{1}}{(0.29,0.30)}, \frac{\mathfrak{y}_{2}}{(0.70,0.60)}, \frac{\mathfrak{y}_{3}}{(0.20,0.50)}, \frac{\mathfrak{y}_{4}}{(0.33,0.40)}\right\}\right\rangle, \\
& \left\langle\left(\frac{0.3}{\mathfrak{S}_{3}}, \mathfrak{e}_{1}, 1\right),\left\{\frac{\mathfrak{y}_{1}}{(0.69,0.21)}, \frac{\mathfrak{y}_{2}}{(0.71,0.17)}, \frac{\mathfrak{y}_{3}}{(0.72,0.32)}, \frac{\mathfrak{y}_{4}}{(0.73,0.25)}\right\}\right\rangle, \\
& \left\langle\left(\frac{0.8}{\mathfrak{S}_{1}}, \mathfrak{e}_{2}, 1\right),\left\{\frac{\mathfrak{y}_{1}}{(0.29,0.40)}, \frac{\mathfrak{y}_{2}}{(0.30,0.70)}, \frac{\mathfrak{y}_{3}}{(0.16,0.44)}, \frac{\mathfrak{y}_{4}}{(0.29,0.70)}\right\}\right\rangle, \\
& \left\langle\left(\frac{0.9}{\mathfrak{F}_{2}}, \mathfrak{e}_{2}, 1\right),\left\{\frac{\mathfrak{y}_{1}}{(0.50,0.30)}, \frac{\mathfrak{y}_{2}}{(0.55,0.71)}, \frac{\mathfrak{y}_{3}}{(0.50,0.40)}, \frac{\mathfrak{y}_{4}}{(0.60,0.50)}\right\}\right\rangle, \\
& \left\langle\left(\frac{0.8}{\mathfrak{g}_{1}}, \mathfrak{e}_{3}, 0\right),\left\{\frac{\mathfrak{y}_{1}}{(0.40,0.60)}, \frac{\mathfrak{y}_{2}}{(0.40,0.70)}, \frac{\mathfrak{y}_{3}}{(0.42,0.72)}, \frac{\mathfrak{y}_{4}}{(0.48,0.20)}\right\}\right\rangle, \\
& \left\langle\left(\frac{0.9}{\mathfrak{S}_{2}}, \mathfrak{e}_{3}, 1\right),\left\{\frac{\mathfrak{y}_{1}}{(0.60,0.72)}, \frac{\mathfrak{y}_{2}}{(0.59,0.91)}, \frac{\mathfrak{y}_{3}}{(0.70,0.30)}, \frac{\mathfrak{y}_{4}}{(0.66,0.82)}\right\}\right\rangle, \\
& \left.\left\langle\left(\frac{0.3}{\mathfrak{G}_{3}}, \mathfrak{e}_{3}, 1\right),\left\{\frac{\mathfrak{y}_{1}}{(0.11,0.29)}, \frac{\mathfrak{y}_{2}}{(0.31,0.42)}, \frac{\mathfrak{y}_{3}}{(0.12,0.24)}, \frac{\mathfrak{y}_{4}}{(0.11,0.23)}\right\}\right\rangle\right\} .
\end{aligned}
$$

Here, $\forall \mathfrak{z} \in \mathscr{Z}, f_{\mathscr{D}}(\mathfrak{z})$ is a 4 -ROF subset of $g_{\mathscr{K}}(\mathfrak{z})$. Hence $(\mathfrak{f}, \mathscr{Z})_{\mathscr{D}} \subset(\mathfrak{g}, \mathscr{W})_{\mathscr{K}}$.

Definition 7. An agree-FP ${ }^{q}$ ROFSES of $(\mathfrak{f}, \mathscr{Z})_{\mathscr{D}}$ over $\mathscr{Y}$ denoted by $(\mathfrak{f}, \mathscr{Z})_{\mathscr{D}}^{1}$ is the $\mathrm{FP}^{q}$ ROFSES defined as $(\mathfrak{f}, \mathscr{Z})_{\mathscr{D}}^{1}=\left\{\mathfrak{f}_{\mathscr{D}}(\mathfrak{z}): \mathfrak{z} \in \mathscr{D} \times \mathscr{E} \times\{1\}\right\}$.
Example 3. Considering the $\mathrm{FP}^{4} \operatorname{ROFSES}(\mathfrak{f}, \mathscr{Z})_{\mathscr{D}}$ in Example 1 , the associated agree-FP $\mathrm{FP}^{4} \operatorname{ROFSES}(\mathfrak{f}, \mathscr{Z})_{\mathscr{D}}^{1}$ over $\mathscr{Y}$ is

$$
\begin{aligned}
(\mathfrak{f}, \mathscr{Z})_{\mathscr{D}}^{1}= & \left\{\left\langle\left(\frac{0.6}{\mathfrak{g}_{1}}, \mathfrak{e}_{1}, 1\right),\left\{\frac{\mathfrak{y}_{1}}{(0.30,0.60)}, \frac{\mathfrak{y}_{2}}{(0.80,0.70)}, \frac{\mathfrak{y}_{3}}{(0.20,0.10)}, \frac{\mathfrak{y}_{4}}{(0.50,0.20)}\right\}\right\rangle,\right. \\
& \left\langle\left(\frac{0.7}{\mathfrak{g}_{2}}, \mathfrak{e}_{1}, 1\right),\left\{\frac{\mathfrak{y}_{1}}{(0.70,0.90)}, \frac{\mathfrak{y}_{2}}{(0.60,0.10)}, \frac{\mathfrak{y}_{3}}{(0.30,0.20)}, \frac{\mathfrak{y}_{4}}{(0.10,0.40)}\right\}\right\rangle, \\
& \left\langle\left(\frac{0.9}{\mathfrak{g}_{3}}, \mathfrak{e}_{1}, 1\right),\left\{\frac{\mathfrak{y}_{1}}{(0.10,0.50)}, \frac{\mathfrak{y}_{2}}{(0.80,0.10)}, \frac{\mathfrak{y}_{3}}{(0.80,0.20)}, \frac{\mathfrak{y}_{4}}{(0.30,0.80)}\right\}\right\rangle, \\
& \left\langle\left(\frac{0.2}{\mathfrak{g}_{4}}, \mathfrak{e}_{1}, 1\right),\left\{\frac{\mathfrak{y}_{1}}{(0.10,0.20)}, \frac{\mathfrak{y}_{2}}{(0.70,0.30)}, \frac{\mathfrak{y}_{3}}{(0.70,0.40)}, \frac{\mathfrak{y}_{4}}{(0.90,0.60)}\right\}\right\rangle,
\end{aligned}
$$




$$
\begin{aligned}
& \left\langle\left(\frac{0.6}{\mathfrak{g}_{1}}, \mathfrak{e}_{2}, 1\right),\left\{\frac{\mathfrak{y}_{1}}{(0.80,0.60)}, \frac{\mathfrak{y}_{2}}{(0.80,0.70)}, \frac{\mathfrak{y}_{3}}{(0.80,0.50)}, \frac{\mathfrak{y}_{4}}{(0.80,0.60)}\right\}\right\rangle, \\
& \left\langle\left(\frac{0.7}{\mathfrak{g}_{2}}, \mathfrak{e}_{2}, 1\right),\left\{\frac{\mathfrak{y}_{1}}{(0.90,0.16)}, \frac{\mathfrak{y}_{2}}{(0.50,0.15)}, \frac{\mathfrak{y}_{3}}{(0.40,0.14)}, \frac{\mathfrak{y}_{4}}{(0.30,0.14)}\right\}\right\rangle, \\
& \left\langle\left(\frac{0.9}{\mathfrak{g}_{3}}, \mathfrak{e}_{2}, 1\right),\left\{\frac{\mathfrak{y}_{1}}{(0.66,0.36)}, \frac{\mathfrak{y}_{2}}{(0.44,0.72)}, \frac{\mathfrak{y}_{3}}{(0.48,0.52)}, \frac{\mathfrak{y}_{4}}{(0.36,0.44)}\right\}\right\rangle, \\
& \left.\left\langle\left(\frac{0.2}{\mathfrak{g}_{4}}, \mathfrak{e}_{2}, 1\right),\left\{\frac{\mathfrak{y}_{1}}{(0.87,0.45)}, \frac{\mathfrak{y}_{2}}{(0.45,0.56)}, \frac{\mathfrak{y}_{3}}{(0.79,0.36)}, \frac{\mathfrak{y}_{4}}{(0.62,0.42)}\right\}\right\rangle\right\} .
\end{aligned}
$$

Definition 8. A disagree-FP ${ }^{q}$ ROFSES of $(\mathfrak{f}, \mathscr{X})_{\mathscr{D}}$ over $\mathscr{Y}$ denoted by $(\mathfrak{f}, \mathscr{Z})_{\mathscr{D}}^{0}$ is the $\mathrm{FP}^{q}$ ROFSES defined as $(\mathfrak{f}, \mathscr{Z})_{\mathscr{D}}^{0}=\left\{f_{\mathscr{D}}(\mathfrak{z}): \mathfrak{z} \in \mathscr{D} \times \mathscr{E} \times\{0\}\right\}$.
Example 4. Considering the $\mathrm{FP}^{4}$ ROFSES $(\mathfrak{f}, \mathscr{Z})_{\mathscr{D}}$ in Example 1 , then the related disagree $\mathrm{FP}^{4}$ ROFSES $(\mathfrak{f}, \mathscr{Z})_{\mathscr{D}}^{0}$ over $y$ is

$$
\begin{aligned}
(\mathfrak{f}, \mathscr{E})_{\mathscr{D}}^{0}= & \left\langle\left\langle\left(\frac{0.6}{\mathfrak{g}_{1}}, \mathfrak{e}_{1}, 0\right),\left\{\frac{\mathfrak{y}_{1}}{(0.70,0.40)}, \frac{\mathfrak{y}_{2}}{(0.60,0.30)}, \frac{\mathfrak{y}_{3}}{(0.70,0.40)}, \frac{\mathfrak{y}_{4}}{(0.80,0.20)}\right\}\right\rangle,\right. \\
& \left\langle\left(\frac{0.7}{\mathfrak{g}_{2}}, \mathfrak{e}_{1}, 0\right),\left\{\frac{\mathfrak{y}_{1}}{(0.70,0.10)}, \frac{\mathfrak{y}_{2}}{(0.80,0.60)}, \frac{\mathfrak{y}_{3}}{(0.40,0.14)}, \frac{\mathfrak{y}_{4}}{(0.56,0.65)}\right\}\right\rangle, \\
& \left\langle\left(\frac{0.9}{\mathfrak{S}_{3}}, \mathfrak{e}_{1}, 0\right),\left\{\frac{\mathfrak{y}_{1}}{(0.80,0.70)}, \frac{\mathfrak{y}_{2}}{(0.90,0.60)}, \frac{\mathfrak{y}_{3}}{(0.80,0.20)}, \frac{\mathfrak{y}_{4}}{(0.80,0.30)}\right\}\right\rangle, \\
& \left\langle\left(\frac{0.2}{\mathfrak{S}_{4}}, \mathfrak{e}_{1}, 0\right),\left\{\frac{\mathfrak{y}_{1}}{(0.50,0.15)}, \frac{\mathfrak{y}_{2}}{(0.77,0.63)}, \frac{\mathfrak{y}_{3}}{(0.56,0.56)}, \frac{\mathfrak{y}_{4}}{(0.27,0.31)}\right\}\right\rangle, \\
& \left\langle\left(\frac{0.6}{\mathfrak{g}_{1}}, \mathfrak{e}_{2}, 0\right),\left\{\frac{\mathfrak{y}_{1}}{(0.90,0.60)}, \frac{\mathfrak{y}_{2}}{(0.62,0.42)}, \frac{\mathfrak{y}_{3}}{(0.71,0.81)}, \frac{\mathfrak{y}_{4}}{(0.72,0.44)}\right\}\right\rangle, \\
& \left\langle\left(\frac{0.7}{\mathfrak{S}_{2}}, \mathfrak{e}_{2}, 0\right),\left\{\frac{\mathfrak{y}_{1}}{(0.40,0.14)}, \frac{\mathfrak{y}_{2}}{(0.53,0.61)}, \frac{\mathfrak{y}_{3}}{(0.71,0.81)}, \frac{\mathfrak{y}_{4}}{(0.90,0.16)}\right\}\right\rangle, \\
& \left\langle\left(\frac{0.9}{\mathfrak{g}_{3}}, \mathfrak{e}_{2}, 0\right),\left\{\frac{\mathfrak{y}_{1}}{(0.72,0.42)}, \frac{\mathfrak{y}_{2}}{(0.77,0.63)}, \frac{\mathfrak{y}_{3}}{(0.50,0.80)}, \frac{\mathfrak{y}_{4}}{(0.30,0.80)}\right\}\right\rangle, \\
& \left.\left\langle\left(\frac{0.2}{\mathfrak{g}_{4}}, \mathfrak{e}_{2}, 0\right),\left\{\frac{\mathfrak{y}_{1}}{(0.30,0.80)}, \frac{\mathfrak{y}_{2}}{(0.79,0.36)}, \frac{\mathfrak{y}_{3}}{(0.80,0.70)}, \frac{\mathfrak{y}_{4}}{(0.50,0.16)}\right\}\right\rangle\right\} .
\end{aligned}
$$

Definition 9. Let $(\mathfrak{f}, \mathscr{Z})_{\mathscr{D}}$ be a $\mathrm{FP}^{q}$ ROFSES over $\mathscr{Y}$. Then, the complement of $\mathrm{FP}^{q}$ ROFSES $(\mathfrak{f}, \mathscr{Z})_{\mathscr{D}}$ represented as $(\mathfrak{f}, \mathscr{Z})_{\mathscr{D}}^{c}$ is defined by $(\mathfrak{f}, \mathscr{Z})_{\mathscr{D}}^{c}=\left(f^{c}, \mathscr{Z}\right)_{\mathscr{D}}$ where $\mathscr{Z} \subseteq \mathscr{D}^{c} \times$ $\mathscr{E} \times \mathscr{O}$ and $f_{\mathscr{D}}^{c}$ is given as $f_{\mathscr{D}}^{c}(\mathfrak{z})=c\left(f_{\mathscr{D}}(\mathfrak{z})\right) \forall \mathfrak{z} \in \mathscr{Z}$ such that

$$
f_{\mathscr{D}}^{c}(\mathfrak{z})=\left\{\frac{\mathfrak{y}}{\left(\beta_{\mathfrak{f}}(\mathfrak{z})(\mathfrak{y}), \alpha_{\mathfrak{f}}(\mathfrak{z})(\mathfrak{y})\right)} \mid \mathfrak{y} \in \mathscr{Y}\right\},
$$

with $0 \leq\left(\alpha_{\mathfrak{f}}(\mathfrak{z})(\mathfrak{y})\right)^{q}+\left(\beta_{\mathfrak{f}}(\mathfrak{z})(\mathfrak{y})\right)^{q} \leq 1$, for all $q \in(0, \infty)$. 
Example 5. Let $(\mathfrak{f}, \mathscr{Z})_{\mathscr{D}}$ be the $\mathrm{FP}^{4}$ ROFSES as discussed in Example 1. Then, by the definition of complement for $\mathrm{FP}^{4}$ ROFSES,

$$
\begin{aligned}
& (\mathfrak{f}, \mathscr{Z})_{\mathscr{D}}^{c}=\left\{\left\langle\left(\frac{0.6}{\mathfrak{g}_{1}}, \mathfrak{e}_{1}, 1\right),\left\{\frac{\mathfrak{y}_{1}}{(0.60,0.30)}, \frac{\mathfrak{y}_{2}}{(0.70,0.80)}, \frac{\mathfrak{y}_{3}}{(0.10,0.20)}, \frac{\mathfrak{y}_{4}}{(0.20,0.50)}\right\}\right\rangle,\right. \\
& \left\langle\left(\frac{0.7}{\mathfrak{g}_{2}}, \mathfrak{e}_{1}, 1\right),\left\{\frac{\mathfrak{y}_{1}}{(0.90,0.70)}, \frac{\mathfrak{y}_{2}}{(0.10,0.60)}, \frac{\mathfrak{y}_{3}}{(0.20,0.30)}, \frac{\mathfrak{y}_{4}}{(0.40,0.10)}\right\}\right\rangle, \\
& \left\langle\left(\frac{0.9}{\mathfrak{g}_{3}}, \mathfrak{e}_{1}, 1\right),\left\{\frac{\mathfrak{y}_{1}}{(0.50,0.10)}, \frac{\mathfrak{y}_{2}}{(0.10,0.80)}, \frac{\mathfrak{y}_{3}}{(0.20,0.80)}, \frac{\mathfrak{y}_{4}}{(0.80,0.30)}\right\}\right\rangle, \\
& \left\langle\left(\frac{0.2}{\mathfrak{g}_{4}}, \mathfrak{e}_{1}, 1\right),\left\{\frac{\mathfrak{y}_{1}}{(0.20,0.10)}, \frac{\mathfrak{y}_{2}}{(0.30,0.70)}, \frac{\mathfrak{y}_{3}}{(0.40,0.70)}, \frac{\mathfrak{y}_{4}}{(0.60,0.90)}\right\}\right\rangle, \\
& \left\langle\left(\frac{0.6}{\mathfrak{S}_{1}}, \mathfrak{e}_{2}, 1\right),\left\{\frac{\mathfrak{y}_{1}}{(0.60,0.80)}, \frac{\mathfrak{y}_{2}}{(0.70,0.80)}, \frac{\mathfrak{y}_{3}}{(0.50,0.80)}, \frac{\mathfrak{y}_{4}}{(0.60,0.80)}\right\}\right\rangle, \\
& \left\langle\left(\frac{0.7}{\mathfrak{g}_{2}}, \mathfrak{e}_{2}, 1\right),\left\{\frac{\mathfrak{y}_{1}}{(0.16,0.90)}, \frac{\mathfrak{y}_{2}}{(0.15,0.50)}, \frac{\mathfrak{y}_{3}}{(0.14,0.40)}, \frac{\mathfrak{y}_{4}}{(0.14,0.30)}\right\}\right\rangle, \\
& \left\langle\left(\frac{0.9}{\mathfrak{g}_{3}}, \mathfrak{e}_{2}, 1\right),\left\{\frac{\mathfrak{y}_{1}}{(0.36,0.66)}, \frac{\mathfrak{y}_{2}}{(0.72,0.44)}, \frac{\mathfrak{y}_{3}}{(0.52,0.48)}, \frac{\mathfrak{y}_{4}}{(0.44,0.36)}\right\}\right\rangle, \\
& \left\langle\left(\frac{0.2}{\mathfrak{g}_{4}}, \mathfrak{e}_{2}, 1\right),\left\{\frac{\mathfrak{y}_{1}}{(0.45,0.87)}, \frac{\mathfrak{y}_{2}}{(0.56,0.45)}, \frac{\mathfrak{y}_{3}}{(0.36,0.79)}, \frac{\mathfrak{y}_{4}}{(0.42,0.62)}\right\}\right\rangle, \\
& \left\langle\left(\frac{0.6}{\mathfrak{g}_{1}}, \mathfrak{e}_{1}, 0\right),\left\{\frac{\mathfrak{y}_{1}}{(0.40,0.70)}, \frac{\mathfrak{y}_{2}}{(0.30,0.60)}, \frac{\mathfrak{y}_{3}}{(0.40,0.70)}, \frac{\mathfrak{y}_{4}}{(0.20,0.80)}\right\}\right\rangle, \\
& \left\langle\left(\frac{0.7}{\mathfrak{g}_{2}}, \mathfrak{e}_{1}, 0\right),\left\{\frac{\mathfrak{y}_{1}}{(0.10,0.70)}, \frac{\mathfrak{y}_{2}}{(0.60,0.80)}, \frac{\mathfrak{y}_{3}}{(0.14,0.40)}, \frac{\mathfrak{y}_{4}}{(0.65,0.56)}\right\}\right\rangle, \\
& \left\langle\left(\frac{0.9}{\mathfrak{g}_{3}}, \mathfrak{e}_{1}, 0\right),\left\{\frac{\mathfrak{y}_{1}}{(0.70,0.80)}, \frac{\mathfrak{y}_{2}}{(0.60,0.90)}, \frac{\mathfrak{y}_{3}}{(0.20,0.80)}, \frac{\mathfrak{y}_{4}}{(0.30,0.80)}\right\}\right\rangle, \\
& \left\langle\left(\frac{0.2}{\mathfrak{g}_{4}}, \mathfrak{e}_{1}, 0\right),\left\{\frac{\mathfrak{y}_{1}}{(0.15,0.50)}, \frac{\mathfrak{y}_{2}}{(0.63,0.77)}, \frac{\mathfrak{y}_{3}}{(0.56,0.56)}, \frac{\mathfrak{y}_{4}}{(0.31,0.27)}\right\}\right\rangle, \\
& \left\langle\left(\frac{0.6}{\mathfrak{S}_{1}}, \mathfrak{e}_{2}, 0\right),\left\{\frac{\mathfrak{y}_{1}}{(0.60,0.90)}, \frac{\mathfrak{y}_{2}}{(0.42,0.62)}, \frac{\mathfrak{y}_{3}}{(0.81,0.71)}, \frac{\mathfrak{y}_{4}}{(0.44,0.72)}\right\}\right\rangle, \\
& \left\langle\left(\frac{0.7}{\mathfrak{g}_{2}}, \mathfrak{e}_{2}, 0\right),\left\{\frac{\mathfrak{y}_{1}}{(0.14,0.40)}, \frac{\mathfrak{y}_{2}}{(0.61,0.53)}, \frac{\mathfrak{y}_{3}}{(0.81,0.71)}, \frac{\mathfrak{y}_{4}}{(0.16,0.90)}\right\}\right\rangle, \\
& \left\langle\left(\frac{0.9}{\mathfrak{g}_{3}}, \mathfrak{e}_{2}, 0\right),\left\{\frac{\mathfrak{y}_{1}}{(0.42,0.72)}, \frac{\mathfrak{y}_{2}}{(0.63,0.77)}, \frac{\mathfrak{y}_{3}}{(0.80,0.50)}, \frac{\mathfrak{y}_{4}}{(0.80,0.30)}\right\}\right\rangle, \\
& \left.\left\langle\left(\frac{0.2}{\mathfrak{S}_{4}}, \mathfrak{e}_{2}, 0\right),\left\{\frac{\mathfrak{y}_{1}}{(0.30,0.80)}, \frac{\mathfrak{y}_{2}}{(0.79,0.36)}, \frac{\mathfrak{y}_{3}}{(0.80,0.70)}, \frac{\mathfrak{y}_{4}}{(0.50,0.16)}\right\}\right\rangle\right\} .
\end{aligned}
$$


Proposition 1. Let $(\mathfrak{f}, \mathscr{Z})_{\mathscr{D}}$ be a FP ROFSES over $\mathscr{Y}$; then, $\left((\mathfrak{f}, \mathscr{Z})_{\mathscr{D}}^{c}\right)^{c}=(\mathfrak{f}, \mathscr{Z})_{\mathscr{D}}$.

Proof. Let $(\mathfrak{f}, \mathscr{Z})_{\mathscr{D}}$ be a $\mathrm{FP}^{q}$ ROFSES over $\mathscr{Y}$. Then, by Definition 9, a mapping $\left(f_{\mathscr{D}}^{c}\right)^{c}: \mathscr{Z} \longrightarrow \mathfrak{Q}^{\mathscr{Y}}$ is defined as follows:

$$
\left(f_{\mathscr{D}}^{c}\right)^{c}(\mathfrak{z})=\left(f_{\mathscr{D}}^{c}(\mathfrak{z})\right)^{c}=f_{\mathscr{D}}(\mathfrak{z}),
$$

for all $\mathfrak{z} \in \mathscr{Z}$, where $\mathscr{Z}=D \times \mathscr{E} \times \mathscr{O}$. Thus, it is proved that $\left((\mathfrak{j}, \mathscr{X})_{\mathscr{D}}^{c}\right)^{c}=(\mathfrak{f}, \mathscr{X})_{\mathscr{D}}$.

Definition 10. The union of two $\mathrm{FP}^{q}$ ROFSESs $(\mathfrak{\mathfrak { f }}, \mathscr{Z})_{\mathscr{D}}$ and $(\mathfrak{g}, \mathscr{W})_{\mathscr{K}}$ over $\mathscr{Y}$ denoted as $(\mathfrak{f}, \mathscr{Z})_{\mathscr{D}} \cup(\mathfrak{g}, \mathscr{W})_{\mathscr{K}}$ is the $\mathrm{FP}^{q}$
ROFSES $(\mathscr{P}, \mathscr{Q})_{\mathscr{R}}$, such that $\mathbb{Q}=(\mathscr{R} \times \mathscr{E} \times \mathscr{O})$, where $\mathscr{R}=\mathscr{D} \cup \mathscr{K}$; then, for all $\mathfrak{z} \in \mathbb{Q}, \mathscr{P}_{\mathscr{R}}(\mathfrak{z})$ is given by

$$
\mathscr{P}_{\mathscr{R}}(\mathfrak{z})=\mathfrak{f}_{\mathscr{D}}(\mathfrak{z}) \cup \mathfrak{g}_{\mathscr{K}}(\mathfrak{z}),
$$

where $U$ is simply $q$-ROF union between $q$-ROFSs $\mathfrak{f}_{\mathscr{D}}(\mathfrak{z})$ and $\mathfrak{g}_{\mathscr{K}}(\mathfrak{z})$.

Example 6. Let $\mathscr{Y}=\left\{\mathfrak{y}_{1}, \mathfrak{y}_{2}, \mathfrak{y}_{3}\right\}$ be the universe, and let $\mathscr{E}=$ $\left\{\mathfrak{e}_{1}, \mathfrak{e}_{2}\right\}$ be the set of experts such that $\mathcal{S}_{\mathscr{Z}}=\left\{\mathfrak{g}_{1}, \mathfrak{g}_{2}\right\}$ and $\mathcal{S}_{\mathscr{W}}=\left\{\mathfrak{g}_{1}\right\} \quad$ represent the sets of parameters, and $\mathscr{D}=\left\{\left(0.8 / \mathfrak{G}_{1}\right),\left(0.6 / \mathfrak{S}_{2}\right)\right\}$, and $\mathscr{K}=\left\{0.8 / \mathfrak{S}_{1}\right\}$ represent the weights for the parameters. Suppose that $(\mathfrak{f}, \mathscr{X})_{\mathscr{D}}$ and $(\mathfrak{g}, \mathscr{W})_{\mathscr{K}}$ are $\mathrm{FP}^{4}$ ROFSESs over $\mathscr{Y}$ such that

$$
\begin{aligned}
& (\mathfrak{f}, \mathscr{Z})_{\mathscr{D}}=\left\{\left\langle\left(\frac{0.8}{\mathfrak{S}_{1}}, \mathfrak{e}_{1}, 1\right),\left\{\frac{\mathfrak{y}_{1}}{(0.80,0.60)}, \frac{\mathfrak{y}_{2}}{(0.30,0.14)}, \frac{\mathfrak{y}_{3}}{(0.45,0.56)}\right\}\right\rangle,\right. \\
& \left\langle\left(\frac{0.6}{\mathfrak{J}_{2}}, \mathfrak{e}_{1}, 1\right),\left\{\frac{\mathfrak{y}_{1}}{(0.40,0.50)}, \frac{\mathfrak{y}_{2}}{(0.52,0.62)}, \frac{\mathfrak{y}_{3}}{(0.60,0.42)}\right\}\right\rangle, \\
& \left\langle\left(\frac{0.8}{\mathfrak{g}_{1}}, \mathfrak{e}_{2}, 1\right),\left\{\frac{\mathfrak{y}_{1}}{(0.70,0.40)}, \frac{\mathfrak{y}_{2}}{(0.43,0.52)}, \frac{\mathfrak{y}_{3}}{(0.60,0.75)}\right\}\right\rangle, \\
& \left\langle\left(\frac{0.6}{\mathfrak{g}_{2}}, \mathfrak{e}_{2}, 1\right),\left\{\frac{\mathfrak{y}_{1}}{(0.36,0.25)}, \frac{\mathfrak{y}_{2}}{(0.30,0.80)}, \frac{\mathfrak{y}_{3}}{(0.45,0.51)}\right\}\right\rangle, \\
& \left\langle\left(\frac{0.8}{\mathfrak{g}_{1}}, \mathfrak{e}_{1}, 0\right),\left\{\frac{\mathfrak{y}_{1}}{(0.80,0.10)}, \frac{\mathfrak{y}_{2}}{(0.90,0.60)}, \frac{\mathfrak{y}_{3}}{(0.60,0.60)}\right\}\right\rangle, \\
& \left\langle\left(\frac{0.6}{\mathfrak{g}_{2}}, \mathfrak{e}_{1}, 0\right),\left\{\frac{\mathfrak{y}_{1}}{(0.50,0.36)}, \frac{\mathfrak{y}_{2}}{(0.48,0.66)}, \frac{\mathfrak{y}_{3}}{(0.55,0.22)}\right\}\right\rangle, \\
& \left\langle\left(\frac{0.8}{\mathfrak{g}_{1}}, \mathfrak{e}_{2}, 0\right),\left\{\frac{\mathfrak{y}_{1}}{(0.44,0.75)}, \frac{\mathfrak{y}_{2}}{(0.71,0.16)}, \frac{\mathfrak{y}_{3}}{(0.22,0.51)}\right\}\right\rangle, \\
& \left.\left\langle\left(\frac{0.6}{\mathfrak{g}_{2}}, \mathfrak{e}_{2}, 0\right),\left\{\frac{\mathfrak{y}_{1}}{(0.66,0.16)}, \frac{\mathfrak{y}_{2}}{(0.60,0.50)}, \frac{\mathfrak{y}_{3}}{(0.28,0.19)}\right\}\right\rangle\right\} \\
& (\mathfrak{g}, \mathscr{W})_{\mathscr{K}}=\left\{\left\langle\left(\frac{0.8}{\mathfrak{g}_{1}}, \mathfrak{e}_{1}, 1\right),\left\{\frac{\mathfrak{y}_{1}}{(0.70,0.50)}, \frac{\mathfrak{y}_{2}}{(0.47,0.55)}, \frac{\mathfrak{y}_{3}}{(0.74,0.45)}\right\}\right\rangle,\right. \\
& \left\langle\left(\frac{0.5}{\mathfrak{g}_{1}}, \mathfrak{e}_{2}, 1\right),\left\{\frac{\mathfrak{y}_{1}}{(0.80,0.40)}, \frac{\mathfrak{y}_{2}}{(0.50,0.35)}, \frac{\mathfrak{y}_{3}}{(0.46,0.78)}\right\}\right\rangle, \\
& \left\langle\left(\frac{0.5}{\mathfrak{g}_{1}}, \mathfrak{e}_{1}, 0\right),\left\{\frac{\mathfrak{y}_{1}}{(0.28,0.51)}, \frac{\mathfrak{y}_{2}}{(0.75,0.32)}, \frac{\mathfrak{y}_{3}}{(0.72,0.56)}\right\}\right\rangle, \\
& \left.\left\langle\left(\frac{0.5}{\mathfrak{Z}_{1}}, \mathfrak{e}_{2}, 0\right),\left\{\frac{\mathfrak{y}_{1}}{(0.81,0.33)}, \frac{\mathfrak{y}_{2}}{(0.40,0.25)}, \frac{\mathfrak{y}_{3}}{(0.62,0.44)}\right\}\right\rangle\right\} \text {. }
\end{aligned}
$$

Using Definition 10 , we obtain $(\mathfrak{f}, \mathscr{Z})_{\mathscr{D}} \cup(\mathfrak{g}, \mathscr{W})_{\mathscr{K}}=$ $(\mathscr{P}, \mathcal{Q})_{\mathscr{R}}$ as follows: 


$$
\begin{aligned}
(\mathscr{P}, Q)_{\mathscr{R}}=\{ & \left\langle\left(\frac{0.8}{\mathfrak{g}_{1}}, \mathfrak{e}_{1}, 1\right),\left\{\frac{\mathfrak{y}_{1}}{(0.80,0.50)}, \frac{\mathfrak{y}_{2}}{(0.47,0.14)}, \frac{\mathfrak{y}_{3}}{(0.74,0.45)}\right\}\right\rangle \\
& \left\langle\left(\frac{0.6}{\mathfrak{g}_{2}}, \mathfrak{e}_{1}, 1\right),\left\{\frac{\mathfrak{y}_{1}}{(0.40,0.50)}, \frac{\mathfrak{y}_{2}}{(0.52,0.62)}, \frac{\mathfrak{y}_{3}}{(0.60,0.42)}\right\}\right\rangle \\
& \left\langle\left(\frac{0.8}{\mathfrak{g}_{1}}, \mathfrak{e}_{2}, 1\right),\left\{\frac{\mathfrak{y}_{1}}{(0.80,0.40)}, \frac{\mathfrak{y}_{2}}{(0.50,0.35)}, \frac{\mathfrak{y}_{3}}{(0.60,0.75)}\right\}\right\rangle \\
& \left\langle\left(\frac{0.6}{\mathfrak{g}_{2}}, \mathfrak{e}_{2}, 1\right),\left\{\frac{\mathfrak{y}_{1}}{(0.36,0.25)}, \frac{\mathfrak{y}_{2}}{(0.30,0.80)}, \frac{\mathfrak{y}_{3}}{(0.45,0.51)}\right\}\right\rangle \\
& \left\langle\left(\frac{0.8}{\mathfrak{g}_{1}}, \mathfrak{e}_{1}, 0\right),\left\{\frac{\mathfrak{y}_{1}}{(0.80,0.10)}, \frac{\mathfrak{y}_{2}}{(0.90,0.32)}, \frac{\mathfrak{y}_{3}}{(0.72,0.56)}\right\}\right\rangle \\
& \left\langle\left(\frac{0.6}{\mathfrak{g}_{2}}, \mathfrak{e}_{1}, 0\right),\left\{\frac{\mathfrak{y}_{1}}{(0.50,0.36)}, \frac{\mathfrak{y}_{2}}{(0.48,0.66)}, \frac{\mathfrak{y}_{3}}{(0.55,0.22)}\right\}\right\rangle \\
& \left\langle\left(\frac{0.8}{\mathfrak{g}_{1}}, \mathfrak{e}_{2}, 0\right),\left\{\frac{\mathfrak{y}_{1}}{(0.81,0.33)}, \frac{\mathfrak{y}_{2}}{(0.71,0.16)}, \frac{\mathfrak{y}_{3}}{(0.62,0.44)}\right\}\right\rangle \\
& \left.\left\langle\left(\frac{0.6}{\mathfrak{F}_{2}}, \mathfrak{e}_{2}, 0\right),\left\{\frac{\mathfrak{y}_{1}}{(0.66,0.16)}, \frac{\mathfrak{y}_{2}}{(0.60,0.50)}, \frac{\mathfrak{y}_{3}}{(0.28,0.19)}\right\}\right\rangle\right\}
\end{aligned}
$$

Proposition 2. Let $(\mathfrak{f}, \mathscr{Z})_{\mathscr{D}},(\mathfrak{g}, \mathscr{W})_{\mathscr{K}}$, and $(\mathfrak{h}, \mathscr{T})_{\mathscr{L}}$ are $F P^{q}$ ROFSESs over the universe $\mathcal{Y}$; then, the following conditions hold:

(1) $(\mathfrak{f}, \mathscr{Z})_{\mathscr{D}} \cup(\mathfrak{g}, \mathscr{W})_{\mathscr{K}}=(\mathfrak{g}, \mathscr{W})_{\mathscr{K}} \cup(\mathfrak{f}, \mathscr{Z})_{\mathscr{D}}$

(2) $\left((\mathfrak{f}, \mathscr{X})_{\mathscr{D}} \cup(\mathfrak{g}, \mathscr{W})_{\mathscr{K}}\right) \cup(\mathfrak{h}, \mathscr{T})_{\mathscr{L}}=(\mathfrak{f}, \mathscr{Z})_{\mathscr{D}} \cup$ $\left((\mathfrak{g}, \mathscr{W})_{\mathscr{K}} \cup(\mathfrak{h}, \mathscr{T})_{\mathscr{L}}\right)$

(3) $(\mathfrak{f}, \mathscr{Z})_{\mathscr{D}} \cup(\mathfrak{f}, \mathscr{X})_{\mathscr{D}}=(\mathfrak{f}, \mathscr{Z})_{\mathscr{D}}$

Proof. (1) Let $(\mathfrak{f}, \mathscr{Z})_{\mathscr{D}} \cup(\mathfrak{g}, \mathscr{W})_{\mathscr{K}}=(\mathscr{P}, \mathscr{Q})_{\mathscr{R}}$; then, $\forall \mathfrak{z}$, we have

$$
\begin{aligned}
\mathscr{R} & =\mathscr{D} \cup \mathscr{K}=\mathscr{K} \cup \mathscr{D}, \\
\mathscr{P}_{\mathscr{R}}(\mathfrak{z}) & =\mathfrak{f}_{\mathscr{D}}(\mathfrak{z}) \cup \mathfrak{g}_{\mathscr{K}}(\mathfrak{z})=\mathfrak{g}_{\mathscr{K}}(\mathfrak{z}) \cup \mathfrak{f}_{\mathscr{D}}(\mathfrak{z}) .
\end{aligned}
$$

This implies $(\mathscr{P}, \mathscr{Q})_{\mathscr{R}}=(\mathfrak{g}, \mathscr{W})_{\mathscr{K}} \cup(\mathfrak{f}, \mathscr{Z})_{\mathscr{D}}$. Hence, $(\mathfrak{f}, \mathscr{Z})_{\mathscr{D}} \cup(\mathfrak{g}, \mathscr{W})_{\mathscr{K}}=(\mathfrak{g}, \mathscr{W})_{\mathscr{K}} \cup(\mathfrak{f}, \mathscr{Z})_{\mathscr{D}}$.
The proof of the remaining parts is similar.

Definition 11. The intersection of two $\mathrm{FP}^{q}$ ROFSESs $(\mathfrak{f}, \mathscr{Z})_{\mathscr{D}}$ and $(\mathfrak{g}, \mathscr{W})_{\mathscr{K}}$ over $\mathscr{Y}$, represented as $(\mathfrak{f}, \mathscr{Z})_{\mathscr{D}} \bar{\cap}(\mathfrak{g}, \mathscr{W})_{\mathscr{K}}$, is the $\operatorname{FP}^{q}$ ROFSESs $(\mathscr{I}, \mathscr{Q})$ such that $\mathscr{Q}=(\mathscr{R} \times \mathscr{E} \times \mathscr{O})$ where $\mathscr{R}=\mathscr{D} \cap \mathscr{K}$. Then, for all $\mathfrak{z} \in \mathscr{Q}, \mathscr{I}_{\mathscr{R}}(\mathfrak{z})$ is defined as

$$
\mathscr{I}_{\mathscr{R}}(\mathfrak{z})=\mathfrak{f}_{\mathscr{D}}(\mathfrak{z}) \cap \mathfrak{g}_{\mathscr{K}}(\mathfrak{z})
$$

where $\cap$ is simply the $q$-ROF intersection between $q$-ROFSs $\mathfrak{f}_{\mathscr{D}}(\mathfrak{z})$ and $\mathfrak{g}_{\mathscr{K}}(\mathfrak{z})$.

Example 7. Let $(\mathfrak{f}, \mathscr{Z})_{\mathscr{D}}$ and $(\mathfrak{g}, \mathscr{W})_{\mathscr{K}}$ be two $\mathrm{FP}^{4}$ ROFSESs over the universe $\mathcal{Y}$, as taken in Example 6. Then, by using Definition 11 , we obtain $(\mathfrak{f}, \mathscr{Z})_{\mathscr{D}} \bar{\cap}(\mathfrak{g}, \mathscr{W})_{\mathscr{K}}=(\mathscr{J}, \mathscr{Q})_{\mathscr{R}}$ where $(\mathscr{I}, \mathscr{Q})_{\mathscr{R}}$ is a $\mathrm{FP}^{4}$ ROFSES defined as

$$
\begin{aligned}
(\mathscr{I}, \mathscr{Q})_{\mathscr{R}}= & \left\{\left\langle\left(\frac{0.8}{\mathfrak{g}_{1}}, \mathfrak{e}_{1}, 1\right),\left\{\frac{\mathfrak{y}_{1}}{(0.70,0.60)}, \frac{\mathfrak{y}_{2}}{(0.30,0.55)}, \frac{\mathfrak{y}_{3}}{(0.45,0.56)}\right\}\right\rangle,\right. \\
& \left\langle\left(\frac{0.6}{\mathfrak{g}_{2}}, \mathfrak{e}_{1}, 1\right),\left\{\frac{\mathfrak{y}_{1}}{(0.40,0.50)}, \frac{\mathfrak{y}_{2}}{(0.52,0.62)}, \frac{\mathfrak{y}_{3}}{(0.60,0.42)}\right\}\right\rangle, \\
& \left\langle\left(\frac{0.8}{\mathfrak{g}_{1}}, \mathfrak{e}_{2}, 1\right),\left\{\frac{\mathfrak{y}_{1}}{(0.70,0.40)}, \frac{\mathfrak{y}_{2}}{(0.43,0.52)}, \frac{\mathfrak{y}_{3}}{(0.46,0.78)}\right\}\right\rangle,
\end{aligned}
$$




$$
\begin{aligned}
& \left\langle\left(\frac{0.6}{\mathfrak{g}_{2}}, \mathfrak{e}_{2}, 1\right),\left\{\frac{\mathfrak{y}_{1}}{(0.36,0.25)}, \frac{\mathfrak{y}_{2}}{(0.30,0.80)}, \frac{\mathfrak{y}_{3}}{(0.45,0.51)}\right\}\right\rangle, \\
& \left\langle\left(\frac{0.8}{\mathfrak{g}_{1}}, \mathfrak{e}_{1}, 0\right),\left\{\frac{\mathfrak{y}_{1}}{(0.28,0.51)}, \frac{\mathfrak{y}_{2}}{(0.75,0.60)}, \frac{\mathfrak{y}_{3}}{(0.60,0.60)}\right\}\right\rangle, \\
& \left\langle\left(\frac{0.6}{\mathfrak{g}_{2}}, \mathfrak{e}_{1}, 0\right),\left\{\frac{\mathfrak{y}_{1}}{(0.50,0.36)}, \frac{\mathfrak{y}_{2}}{(0.48,0.66)}, \frac{\mathfrak{y}_{3}}{(0.55,0.22)}\right\}\right\rangle, \\
& \left\langle\left(\frac{0.8}{\mathfrak{g}_{1}}, \mathfrak{e}_{2}, 0\right),\left\{\frac{\mathfrak{y}_{1}}{(0.44,0.75)}, \frac{\mathfrak{y}_{2}}{(0.40,0.25)}, \frac{\mathfrak{y}_{3}}{(0.22,0.51)}\right\}\right\rangle, \\
& \left.\left\langle\left(\frac{0.6}{\mathfrak{g}_{2}}, \mathfrak{e}_{2}, 0\right),\left\{\frac{\mathfrak{y}_{1}}{(0.66,0.16)}, \frac{\mathfrak{y}_{2}}{(0.60,0.50)}, \frac{\mathfrak{y}_{3}}{(0.28,0.19)}\right\}\right\rangle\right\} .
\end{aligned}
$$

Proposition 3. Let $(\mathfrak{f}, \mathscr{Z})_{\mathscr{D}},(\mathfrak{g}, \mathscr{W})_{k}$, and $(\mathfrak{h}, \mathscr{T})_{\mathscr{L}}$ be an $F^{q}$ ROFSES over the universe $\mathcal{Y}$; then, the following conditions hold:

(1) $(\mathfrak{f}, \mathscr{Z})_{\mathscr{D}} \bar{\cap}(\mathfrak{g}, \mathscr{W})_{\mathscr{K}}=(\mathfrak{g}, \mathscr{W})_{\mathscr{K}} \bar{\cap}(\mathfrak{f}, \mathscr{Z})_{\mathscr{D}}$

(2) $\left((\mathfrak{f}, \mathscr{Z})_{\mathscr{D}} \overline{\mathrm{n}} \quad(\mathfrak{g}, \mathscr{W})_{\mathscr{K}}\right) \bar{\cap}(\mathfrak{h}, \mathscr{T})_{\mathscr{L}}=$ $(\mathfrak{f}, \mathscr{Z})_{\mathscr{D}} \bar{\cap}\left((\mathfrak{g}, \mathscr{W})_{\mathscr{K}} \bar{\cap}(\mathfrak{h}, \mathscr{T})_{\mathscr{L}}\right)$

(3) $(\mathfrak{f}, \mathscr{Z})_{\mathscr{D}} \bar{\cap}(\mathfrak{f}, \mathscr{Z})_{\mathscr{D}}=(\mathfrak{f}, \mathscr{Z})_{\mathscr{D}}$

Proof. (1) Let $(\mathfrak{f}, \mathscr{Z})_{\mathscr{D}} \bar{\cap}(\mathfrak{g}, \mathscr{W})_{\mathscr{K}}=(\mathscr{I}, \mathscr{Q})_{\mathscr{R}}$; then, $\forall \mathfrak{z} \in \mathbb{Q}$, we have

$$
\begin{aligned}
\mathscr{R} & =\mathscr{D} \cap \mathscr{K}=\mathscr{K} \cap \mathscr{D} \\
\mathscr{I}_{\mathscr{R}}(\mathfrak{z}) & =\mathfrak{f}_{\mathscr{D}}(\mathfrak{z}) \cap \mathfrak{g}_{\mathscr{K}}(\mathfrak{z})=\mathfrak{g}_{\mathscr{K}}(\mathfrak{z}) \cap \mathfrak{f}_{\mathscr{D}}(\mathfrak{z}) .
\end{aligned}
$$

This implies $(\mathscr{I}, \mathscr{Q})_{\mathscr{R}}=(\mathfrak{g}, \mathscr{W})_{\mathscr{K}} \bar{\cap}(\mathfrak{f}, \mathscr{Z})_{\mathscr{D}}$. Hence, $(\mathfrak{f}, \mathscr{Z})_{\mathscr{D}} \bar{\cap}(\mathfrak{g}, \mathscr{W})_{\mathscr{K}}=(\mathfrak{g}, \mathscr{W})_{\mathscr{K}} \bar{\cap}(\mathfrak{f}, \mathscr{Z})_{\mathscr{D}}$.

The proof of the remaining parts is similar.

Proposition 4. Let $(\mathfrak{f}, \mathscr{Z})_{\mathscr{D}},(\mathfrak{g}, \mathscr{W})_{\mathscr{K}}$, and $(\mathfrak{h}, \mathscr{T})_{\mathscr{L}}$ be FPq ROFSESs over the universe $\mathscr{Y}$; then, the following conditions hold:

$$
\begin{array}{ll}
\text { (1) } & (\mathfrak{f}, \mathscr{Z})_{\mathscr{D}} \bar{\cap}\left((\mathfrak{g}, \mathscr{W})_{\mathscr{K}} \cup \quad(\mathfrak{h}, \mathscr{T})_{\mathscr{L}}\right)=\left((\mathfrak{f}, \mathscr{Z})_{\mathscr{D}}\right. \\
& \left.\bar{\cap}(\mathfrak{g}, \mathscr{W})_{\mathscr{K}}\right) \cup\left((\mathfrak{f}, \mathscr{Z})_{\mathscr{D}} \bar{\cap}(\mathfrak{h}, \mathscr{T})_{\mathscr{L}}\right) \\
\text { (2) } & (\mathfrak{f}, \mathscr{Z})_{\mathscr{D}} \cup\left((\mathfrak{g}, \mathscr{W})_{\mathscr{K}} \bar{\cap}(\mathfrak{h}, \mathscr{T})_{\mathscr{L}}\right)= \\
& \left((\mathfrak{f}, \mathscr{Z})_{\mathscr{D}} \cup(\mathfrak{g}, \mathscr{W})_{\mathscr{K}}\right) \bar{\cap}\left((\mathfrak{f}, \mathscr{Z})_{\mathscr{D}} \cup(\mathfrak{h}, \mathscr{T})_{\mathscr{L}}\right)
\end{array}
$$

Proof. The proof is simple and is therefore omitted.

Proposition 5. Let $(\mathfrak{f}, \mathscr{Z})_{\mathscr{D}}$ and $(\mathfrak{g}, \mathscr{W})_{\mathscr{K}}$ be FPq ROFSESs over the universe $\mathcal{Y}$. Then,

$$
\begin{aligned}
& \text { (1) }\left((\mathfrak{f}, \mathscr{Z})_{\mathscr{D}} \cup(\mathfrak{g}, \mathscr{W})_{\mathscr{K}}\right)^{c}=(\mathfrak{f}, \mathscr{Z})_{\mathscr{D}}^{c} \bar{\cap}(\mathfrak{g}, \mathscr{W})_{\mathscr{K}}^{c} \\
& \text { (2) }\left((\mathfrak{f}, \mathscr{Z})_{\mathscr{D}} \bar{\cap}(\mathfrak{g}, \mathscr{W})_{\mathscr{K}}\right)^{c}=(\mathfrak{f}, \mathscr{Z})_{\mathscr{D}}^{c} \cup(\mathfrak{g}, \mathscr{W})_{\mathscr{K}}^{c}
\end{aligned}
$$

Proof. (1) Let $(\mathfrak{f}, \mathscr{Z})_{\mathscr{D}}$ and $(\mathfrak{g}, \mathscr{W})_{\mathscr{K}}$ be two FP ROFSESs over the universe $\mathscr{Y}$; then, we have

$$
\mathfrak{f}_{\mathscr{D}}^{c}(\mathfrak{z}) \cap \mathfrak{g}_{\mathscr{K}}^{c}(\mathfrak{z})=\left(\mathfrak{f}_{\mathscr{D}}(\mathfrak{z}) \cup \mathfrak{g}_{\mathscr{K}}(\mathfrak{z})\right)^{c},
$$

for all $\mathfrak{z} \in \mathscr{D} \cap \mathscr{K}$. Hence, $\left((\mathfrak{f}, \mathscr{Z})_{\mathscr{D}} \cup(\mathfrak{g}, \mathscr{W})_{\mathscr{K}}\right)^{c}=(\mathfrak{f}, \mathscr{Z})_{\mathscr{D}}^{c}$ $\cap(\mathfrak{g}, \mathscr{W})_{\mathscr{K}}^{c}$.

(2) Similar to Part 1.

Definition 12. Let $(\mathfrak{f}, \mathscr{Z})_{\mathscr{D}}$ and $(\mathfrak{g}, \mathscr{W})_{\mathscr{K}}$ be two FP ${ }^{q}$ ROFSESs over the universe $\mathscr{Y}$. Then, " $\left((\mathfrak{f}, \mathscr{Z})_{\mathscr{D}} \mathrm{AND}(\mathfrak{g}, \mathscr{W})_{\mathscr{K}}\right)$ " represented by $(\mathfrak{f}, \mathscr{Z})_{\mathscr{D}} \wedge(\mathfrak{g}, \mathscr{W})_{\mathscr{K}}=(\mathfrak{I}, \mathscr{Z} \times \mathscr{W})_{\mathscr{R}}$ is an $\mathrm{FP}^{q}$ ROFSES such that $\mathscr{R}=\mathscr{D} \times \mathscr{K}$ and is given by

$$
\mathfrak{\Im}_{\mathscr{R}}(\sigma, \delta)=\left(\mathfrak{f}_{\mathscr{D}}(\sigma) \cap \mathfrak{g}_{\mathscr{K}}(\delta)\right), \quad \forall(\sigma, \delta) \in \mathscr{Z} \times \mathscr{W} .
$$

Definition 13. Let $(\mathfrak{f}, \mathscr{Z})_{\mathscr{D}}$ and $(\mathfrak{g}, \mathscr{W})_{\mathscr{K}}$ be two $\mathrm{FP}^{q} \mathrm{ROF}-$ SESs over the universe $\mathscr{Y}$. Then, " $\left((\mathfrak{f}, \mathscr{Z})_{\mathscr{D}} \mathrm{OR}(\mathfrak{g}, \mathscr{W})_{\mathscr{K}}\right)$ " represented by $(\mathfrak{f}, \mathscr{Z})_{\mathscr{D}} \vee(\mathfrak{g}, \mathscr{W})_{\mathscr{K}}=(\mathfrak{P}, \mathscr{Z} \times \mathscr{W})_{\mathscr{R}}$ is an $\mathrm{FP}^{q}$ ROFSES such that $\mathscr{R}=\mathscr{D} \times \mathscr{K}$ and is given as

$$
\mathfrak{P}_{\mathscr{R}}(\sigma, \delta)=\left(\mathfrak{f}_{\mathscr{D}}(\sigma) \cup \mathfrak{g}_{\mathscr{K}}(\delta)\right), \quad \forall(\sigma, \delta) \in \mathscr{Z} \times \mathscr{W}
$$

Proposition 6. Let $(\mathfrak{f}, \mathscr{Z})_{\mathscr{D}}$ and $(\mathfrak{g}, \mathscr{W})_{\mathscr{K}}$ be FP $P^{q}$ ROFSESs over the universe $\mathcal{Y}$. Then,

$$
\begin{aligned}
& \text { (1) }\left((\mathfrak{f}, \mathscr{Z})_{\mathscr{D}} \wedge(\mathfrak{g}, \mathscr{W})_{\mathscr{K}}\right)^{c}=(\mathfrak{f}, \mathscr{Z})_{\mathscr{D}}^{c} \vee(\mathfrak{g}, \mathscr{W})_{\mathscr{K}}^{c} \\
& \text { (2) }\left((\mathfrak{f}, \mathscr{Z})_{\mathscr{D}} \vee(\mathfrak{g}, \mathscr{W})_{\mathscr{K}}\right)^{c}=(\mathfrak{f}, \mathscr{Z})_{\mathscr{D}}^{c} \wedge(\mathfrak{g}, \mathscr{W})_{\mathscr{K}}^{c}
\end{aligned}
$$

Proof. Its proof is directly followed from Proposition 5.

\section{Applications of $\mathbf{F P}^{q}$ ROFSESs in Group Decision-Making}

As a powerful extension to the existing fuzzy parameterized models, $\mathrm{FP}^{q}$ ROFSESs are highly applicable and can prove their efficiency in situations where other models failed. This section provides two MAGDM problems and their solutions under $\mathrm{FP}^{q}$ ROFSESs along with a developed algorithm.

Before going through the applications, some notions need to be reviewed as follows:

Definition 14 (see [9]). Let $\mathbb{Q}=\left(\alpha_{\mathfrak{f}}\left(\mathfrak{z}_{i}\right)\left(\mathfrak{y}_{j}\right), \beta_{\mathfrak{f}}\left(\mathfrak{z}_{i}\right)\left(\mathfrak{y}_{j}\right)\right)$ be a $q$-ROFN. The score function of $Q$ is given by

$$
s(\mathscr{Q})=\left(\alpha_{\mathfrak{f}}\left(\mathfrak{z}_{i}\right)\left(\mathfrak{y}_{j}\right)\right)^{q}-\left(\beta_{\mathfrak{f}}\left(\mathfrak{z}_{i}\right)\left(\mathfrak{y}_{j}\right)\right)^{q}, \quad q \geq 1 .
$$


Definition 15 (see [9]). Let $Q=\left(\alpha_{\mathfrak{f}}\left(\mathfrak{z}_{i}\right)\left(\mathfrak{y}_{j}\right), \beta_{\mathfrak{f}}\left(\mathfrak{z}_{i}\right)\left(\mathfrak{y}_{j}\right)\right)$ be a $q$-ROFN. The accuracy function of $Q$ is given as

$$
h(\mathscr{Q})=\left(\alpha_{\mathfrak{f}}\left(\mathfrak{z}_{i}\right)\left(\mathfrak{y}_{j}\right)\right)^{q}+\left(\beta_{\mathfrak{f}}\left(\mathfrak{z}_{i}\right)\left(\mathfrak{y}_{j}\right)\right)^{q}, \quad q \geq 1 .
$$

Definition 16 (see [9]). Let $\mathbb{Q}_{1}=\left(\alpha_{\mathfrak{f}_{1}}\left(\mathfrak{z}_{i}\right)\left(\mathfrak{y}_{j}\right), \beta_{\mathfrak{f}_{1}}\left(\mathfrak{z}_{i}\right)\left(\mathfrak{y}_{j}\right)\right)$ and $Q_{2}=\left(\alpha_{\mathfrak{f}_{2}}\left(z_{i}\right)\left(\mathfrak{y}_{j}\right), \beta_{\mathfrak{f}_{2}}\left(z_{i}\right)\left(\mathfrak{y}_{j}\right)\right)$ be any two $q$-ROFNs, let $s\left(Q_{1}\right)$ and $s\left(Q_{2}\right)$ be the score functions of $Q_{1}$ and $Q_{2}$, and let $h\left(Q_{1}\right)$ and $h\left(Q_{2}\right)$ be the accuracy functions of $Q_{1}$ and $Q_{2}$; then,

(1) If $s\left(Q_{1}\right)>s\left(Q_{2}\right)$, then $Q_{1}>Q_{2}$

(2) If $s\left(\mathbb{Q}_{1}\right)=s\left(\mathbb{Q}_{2}\right)$

(i) If $h\left(Q_{1}\right)>h\left(Q_{2}\right)$, then $Q_{1}>Q_{2}$

(ii) If $h\left(Q_{1}\right)=h\left(Q_{2}\right)$, then $Q_{1}=\mathbb{Q}_{2}$

Definition 17. For an agree-FP ${ }^{q} \operatorname{ROFSES}(\mathfrak{f}, \mathscr{Z})_{\mathscr{D}}^{1}$, its accumulated agree scores $L_{j}$ are given as

$$
L_{j}=\sum_{i} w_{i} X_{i j}
$$

where $X_{i j}$ 's are entries of the score table for agree-FP $q$ ROFSES and $w_{i}$ represents weights for the parameters.

Definition 18. For a disagree-FP $\mathrm{FP}^{q} \operatorname{ROFES}(\mathfrak{f}, \mathscr{Z})_{\mathscr{D}}^{0}$, its accumulated disagree scores $C_{j}$ are given by

$$
C_{j}=\sum_{i} w_{i} X_{i j}
$$

where $X_{i j}$ 's are entries of the score table for disagree-FPq ROFSES and $w_{i}$ represents weights for the parameters.

Definition 19. For a $\operatorname{FP}^{q} \operatorname{ROFSES}(\mathfrak{f}, \mathscr{Z})_{\mathscr{D}}$, its final scores $\mathfrak{\Re}_{j}$ are defined as

$$
\mathfrak{\Re}_{j}=L_{j}-C_{j} .
$$

We now present the applications of $\mathrm{FP}^{q}$ ROFSESs in the following.

\subsection{Selection of an Appropriate Site for a Cafe Outlet.}

Cafes are considered as places where one can meet up with his friends, sit away from home and work, or just simply grab a cup of coffee and let the laziness go away. Cafes are now present all over the world, and those owning these cafes are making huge profits all around. The cafe and restaurant business comprises a large part of the economy and is considered a catchy business to make a career with. Many cafe chains like McDonald's McCafe, Starbucks, Tim Hortons, etc., are well known for their worldwide business and reputation. Similarly, many small cafe chains are also reputable for the quality and services they provide.

The cafe chains keep on earning, growing, expanding, and again earning more. But many factors affect the cafe business, particularly where the cafe outlet is located. If the cafe is in a good place with many customers and less competition, then, it assures a good business if ran professionally. On the other hand, if the cafe is in a place with fewer customers or a high competition place, then, it can prove to be a challenge for the owners to make a good profit from it. In the example below, we are going to see how $\mathrm{FP}^{q}$ ROFSESs can help in finding the best location for a cafe outlet, so that the business gets profited instead of falling back.

Consider a cafe chain is willing to increase its business by opening a new outlet in the area of the city, where the chain has no prior outlets. To get the best choice, the chain considers experts' opinions in the selection process of an appropriate site for their new outlet. The cafe chain owner has five sites under consideration to extend their chain. Let $\mathscr{Y}=\left\{\mathfrak{y}_{1}, \mathfrak{y}_{2}, \ldots, \mathfrak{y}_{5}\right\}$ show the set of sites available. The experts consider $\mathcal{S}=\left\{\mathfrak{G}_{1}, \mathfrak{g}_{2}, \mathfrak{g}_{3}\right\}$ as the set of important parameters for the analysis of the sites, where $\mathfrak{g}_{1}$ is the competing cafes within the chosen area, $\mathfrak{g}_{2}$ is the fraction of residents who visit one cafe for coffee or tea, and $\mathfrak{g}_{3}$ is the cafe's accessibility which includes ample parking. Consider that $\mathscr{E}=\left\{\mathfrak{e}_{1}, \mathfrak{e}_{2}, \mathfrak{e}_{3}, \mathfrak{e}_{4}\right\}$ is the set of experts and $\mathscr{D}=\left\{\left(0.5 / \mathfrak{g}_{1}\right),\left(0.3 / \mathfrak{g}_{2}\right),\left(0.8 / \mathfrak{g}_{3}\right)\right\}$ represents the weights for the parameters.

The experts visit and analyze the sites with respect to the indicated parameters and make their findings integrated into an $\mathrm{FP}^{q}$ ROFSES where $q=4$ as shown as follows:

$$
\begin{aligned}
(\mathfrak{f}, \mathscr{Z})_{\mathscr{D}}= & \left\{\left\langle\left(\frac{0.5}{\mathfrak{g}_{1}}, \mathfrak{e}_{1}, 1\right)\left\{\frac{\mathfrak{y}_{1}}{(0.90,0.70)}, \frac{\mathfrak{y}_{2}}{(0.42,0.38)}, \frac{\mathfrak{y}_{3}}{(0.11,0.61)}, \frac{\mathfrak{y}_{4}}{(0.80,0.70)}, \frac{\mathfrak{y}_{5}}{(0.58,0.47)}\right\}\right\rangle,\right. \\
& \left\langle\left(\frac{0.3}{\mathfrak{g}_{2}}, \mathfrak{e}_{1}, 1\right)\left\{\frac{\mathfrak{y}_{1}}{(0.24,0.39)}, \frac{\mathfrak{y}_{2}}{(0.19,0.28)}, \frac{\mathfrak{y}_{3}}{(0.43,0.16)}, \frac{\mathfrak{y}_{4}}{(0.53,0.37)}, \frac{\mathfrak{y}_{5}}{(0.69,0.36)}\right\}\right\rangle, \\
& \left\langle\left(\frac{0.8}{\mathfrak{g}_{3}}, \mathfrak{e}_{1}, 1\right)\left\{\frac{\mathfrak{y}_{1}}{(0.29,0.37)}, \frac{\mathfrak{y}_{2}}{(0.89,0.70)}, \frac{\mathfrak{y}_{3}}{(0.83,0.20)}, \frac{\mathfrak{y}_{4}}{(0.14,0.25)}, \frac{\mathfrak{y}_{5}}{(0.39,0.75)}\right\}\right\rangle, \\
& \left\langle\left(\frac{0.5}{\mathfrak{g}_{1}}, \mathfrak{e}_{2}, 1\right)\left\{\frac{\mathfrak{y}_{1}}{(0.51,0.75)}, \frac{\mathfrak{y}_{2}}{(0.45,0.65)}, \frac{\mathfrak{y}_{3}}{(0.12,0.78)}, \frac{\mathfrak{y}_{4}}{(0.56,0.72)}, \frac{\mathfrak{y}_{5}}{(0.90,0.43)}\right\}\right\rangle,
\end{aligned}
$$




$$
\begin{aligned}
& \left\langle\left(\frac{0.3}{\mathfrak{g}_{2}}, \mathfrak{e}_{2}, 1\right)\left\{\frac{\mathfrak{y}_{1}}{(0.27,0.45)}, \frac{\mathfrak{y}_{2}}{(0.21,0.27)}, \frac{\mathfrak{y}_{3}}{(0.25,0.27)}, \frac{\mathfrak{y}_{4}}{(0.69,0.15)}, \frac{\mathfrak{y}_{5}}{(0.34,0.13)}\right\}\right\rangle, \\
& \left\langle\left(\frac{0.8}{\mathfrak{Z}_{3}}, \mathfrak{e}_{2}, 1\right)\left\{\frac{\mathfrak{y}_{1}}{(0.44,0.81)}, \frac{\mathfrak{y}_{2}}{(0.45,0.65)}, \frac{\mathfrak{y}_{3}}{(0.32,0.41)}, \frac{\mathfrak{y}_{4}}{(0.29,0.23)}, \frac{\mathfrak{y}_{5}}{(0.88,0.34)}\right\}\right\rangle, \\
& \left\langle\left(\frac{0.5}{\mathfrak{Z}_{1}}, \mathfrak{e}_{3}, 1\right)\left\{\frac{\mathfrak{y}_{1}}{(0.47,0.62)}, \frac{\mathfrak{y}_{2}}{(0.32,0.34)}, \frac{\mathfrak{y}_{3}}{(0.40,0.36)}, \frac{\mathfrak{y}_{4}}{(0.28,0.42)}, \frac{\mathfrak{y}_{5}}{(0.46,0.16)}\right\}\right\rangle, \\
& \left\langle\left(\frac{0.3}{\mathfrak{g}_{2}}, \mathfrak{e}_{3}, 1\right)\left\{\frac{\mathfrak{y}_{1}}{(0.60,0.80)}, \frac{\mathfrak{y}_{2}}{(0.24,0.47)}, \frac{\mathfrak{y}_{3}}{(0.59,0.37)}, \frac{\mathfrak{y}_{4}}{(0.11,0.27)}, \frac{\mathfrak{y}_{5}}{(0.64,0.20)}\right\}\right\rangle, \\
& \left\langle\left(\frac{0.8}{\mathfrak{Z}_{3}}, \mathfrak{e}_{3}, 1\right)\left\{\frac{\mathfrak{y}_{1}}{(0.81,0.52)}, \frac{\mathfrak{y}_{2}}{(0.56,0.46)}, \frac{\mathfrak{y}_{3}}{(0.22,0.65)}, \frac{\mathfrak{y}_{4}}{(0.92,0.34)}, \frac{\mathfrak{y}_{5}}{(0.37,0.56)}\right\}\right\rangle, \\
& \left\langle\left(\frac{0.5}{\mathfrak{I}_{1}}, \mathfrak{e}_{4}, 1\right)\left\{\frac{\mathfrak{y}_{1}}{(0.72,0.44)}, \frac{\mathfrak{y}_{2}}{(0.56,0.65)}, \frac{\mathfrak{y}_{3}}{(0.63,0.36)}, \frac{\mathfrak{y}_{4}}{(0.43,0.51)}, \frac{\mathfrak{y}_{5}}{(0.30,0.80)}\right\}\right\rangle, \\
& \left\langle\left(\frac{0.3}{\mathfrak{Z}_{2}}, \mathfrak{e}_{4}, 1\right)\left\{\frac{\mathfrak{y}_{1}}{(0.66,0.36)}, \frac{\mathfrak{y}_{2}}{(0.44,0.72)}, \frac{\mathfrak{y}_{3}}{(0.48,0.52)}, \frac{\mathfrak{y}_{4}}{(0.45,0.56)}, \frac{\mathfrak{y}_{5}}{(0.51,0.42)}\right\}\right\rangle, \\
& \left\langle\left(\frac{0.8}{\mathfrak{Z}_{3}}, \mathfrak{e}_{4}, 1\right)\left\{\frac{\mathfrak{y}_{1}}{(0.87,0.45)}, \frac{\mathfrak{y}_{2}}{(0.62,0.42)}, \frac{\mathfrak{y}_{3}}{(0.77,0.63)}, \frac{\mathfrak{y}_{4}}{(0.30,0.14)}, \frac{\mathfrak{y}_{5}}{(0.70,0.21)}\right\}\right\rangle, \\
& \left\langle\left(\frac{0.5}{\mathfrak{I}_{1}}, \mathfrak{e}_{1}, 0\right)\left\{\frac{\mathfrak{y}_{1}}{(0.79,0.50)}, \frac{\mathfrak{y}_{2}}{(0.81,0.27)}, \frac{\mathfrak{y}_{3}}{(0.84,0.50)}, \frac{\mathfrak{y}_{4}}{(0.86,0.40)}, \frac{\mathfrak{y}_{5}}{(0.15,0.22)}\right\}\right\rangle, \\
& \left\langle\left(\frac{0.3}{\mathfrak{F}_{2}}, \mathfrak{e}_{1}, 0\right)\left\{\frac{\mathfrak{y}_{1}}{(0.15,0.22)}, \frac{\mathfrak{y}_{2}}{(0.17,0.25)}, \frac{\mathfrak{y}_{3}}{(0.19,0.22)}, \frac{\mathfrak{y}_{4}}{(0.17,0.21)}, \frac{\mathfrak{y}_{5}}{(0.11,0.24)}\right\}\right\rangle, \\
& \left\langle\left(\frac{0.8}{\mathfrak{S}_{3}}, \mathfrak{e}_{1}, 0\right)\left\{\frac{\mathfrak{y}_{1}}{(0.31,0.42)}, \frac{\mathfrak{y}_{2}}{(0.12,0.24)}, \frac{\mathfrak{y}_{3}}{(0.11,0.23)}, \frac{\mathfrak{y}_{4}}{(0.10,0.22)}, \frac{\mathfrak{y}_{5}}{(0.34,0.46)}\right\}\right\rangle, \\
& \left\langle\left(\frac{0.5}{\mathfrak{Z}_{1}}, \mathfrak{e}_{2}, 0\right)\left\{\frac{\mathfrak{y}_{1}}{(0.37,0.39)}, \frac{\mathfrak{y}_{2}}{(0.18,0.70)}, \frac{\mathfrak{y}_{3}}{(0.13,0.25)}, \frac{\mathfrak{y}_{4}}{(0.38,0.45)}, \frac{\mathfrak{y}_{5}}{(0.35,0.45)}\right\}\right\rangle, \\
& \left\langle\left(\frac{0.3}{\mathfrak{F}_{2}}, \mathfrak{e}_{2}, 0\right)\left\{\frac{\mathfrak{y}_{1}}{(0.34,0.40)}, \frac{\mathfrak{y}_{2}}{(0.39,0.29)}, \frac{\mathfrak{y}_{3}}{(0.38,0.27)}, \frac{\mathfrak{y}_{4}}{(0.15,0.20)}, \frac{\mathfrak{y}_{5}}{(0.51,0.36)}\right\}\right\rangle, \\
& \left\langle\left(\frac{0.8}{\mathfrak{S}_{3}}, \mathfrak{e}_{2}, 0\right)\left\{\frac{\mathfrak{y}_{1}}{(0.54,0.22)}, \frac{\mathfrak{y}_{2}}{(0.13,0.37)}, \frac{\mathfrak{y}_{3}}{(0.55,0.15)}, \frac{\mathfrak{y}_{4}}{(0.62,0.10)}, \frac{\mathfrak{y}_{5}}{(0.45,0.16)}\right\}\right\rangle, \\
& \left\langle\left(\frac{0.5}{\mathfrak{Z}_{1}}, \mathfrak{e}_{3}, 0\right)\left\{\frac{\mathfrak{y}_{1}}{(0.63,0.45)}, \frac{\mathfrak{y}_{2}}{(0.39,0.10)}, \frac{\mathfrak{y}_{3}}{(0.64,0.19)}, \frac{\mathfrak{y}_{4}}{(0.63,0.15)}, \frac{\mathfrak{y}_{5}}{(0.62,0.17)}\right\}\right\rangle, \\
& \left\langle\left(\frac{0.3}{\mathfrak{F}_{2}}, \mathfrak{e}_{3}, 0\right)\left\{\frac{\mathfrak{y}_{1}}{(0.69,0.21)}, \frac{\mathfrak{y}_{2}}{(0.71,0.17)}, \frac{\mathfrak{y}_{3}}{(0.72,0.32)}, \frac{\mathfrak{y}_{4}}{(0.73,0.25)}, \frac{\mathfrak{y}_{5}}{(0.12,0.30)}\right\}\right\rangle, \\
& \left\langle\left(\frac{0.8}{\mathfrak{F}_{3}}, \mathfrak{e}_{3}, 0\right)\left\{\frac{\mathfrak{y}_{1}}{(0.40,0.36)}, \frac{\mathfrak{y}_{2}}{(0.29,0.23)}, \frac{\mathfrak{y}_{3}}{(0.12,0.28)}, \frac{\mathfrak{y}_{4}}{(0.45,0.65)}, \frac{\mathfrak{y}_{5}}{(0.36,0.55)}\right\}\right\rangle, \\
& \left\langle\left(\frac{0.5}{\mathfrak{Z}_{1}}, \mathfrak{e}_{4}, 0\right)\left\{\frac{\mathfrak{y}_{1}}{(0.29,0.37)}, \frac{\mathfrak{y}_{2}}{(0.81,0.75)}, \frac{\mathfrak{y}_{3}}{(0.27,0.43)}, \frac{\mathfrak{y}_{4}}{(0.47,0.62)}, \frac{\mathfrak{y}_{5}}{(0.81,0.52)}\right\}\right\rangle,
\end{aligned}
$$




$$
\begin{aligned}
& \left\langle\left(\frac{0.3}{\mathfrak{g}_{2}}, \mathfrak{e}_{4}, 0\right)\left\{\frac{\mathfrak{y}_{1}}{(0.43,0.16)}, \frac{\mathfrak{y}_{2}}{(0.83,0.70)}, \frac{\mathfrak{y}_{3}}{(0.21,0.27)}, \frac{\mathfrak{y}_{4}}{(0.40,0.36)}, \frac{\mathfrak{y}_{5}}{(0.59,0.37)}\right\}\right\rangle, \\
& \left.\left\langle\left(\frac{0.8}{\mathfrak{g}_{3}}, \mathfrak{e}_{4}, 0\right)\left\{\frac{\mathfrak{y}_{1}}{(0.32,0.41)}, \frac{\mathfrak{y}_{2}}{(0.24,0.47)}, \frac{\mathfrak{y}_{3}}{(0.27,0.25)}, \frac{\mathfrak{y}_{4}}{(0.89,0.60)}, \frac{\mathfrak{y}_{5}}{(0.92,0.34)}\right\}\right\rangle\right\} .
\end{aligned}
$$

For the selection of the best site based on the above data, the experts analyze the data under the decision-making method based on $\mathrm{FP}^{q}$ ROFSESs as given in Algorithm 1.

Table 1 represents the score table for the $\mathrm{FP}^{4}$ ROFSES defined above. Tables 2 and 3 represent the score tables for agree- and disagree- $\mathrm{FP}^{4}$ ROFSESs, respectively, along with the agree and disagree accumulated scores.

Using agree and disagree accumulated scores in Tables 2 and 3, Table 4 provides the final scores. From the final scores table, it can be seen that $\boldsymbol{\Re}_{M}=\max \left(\boldsymbol{K}_{j}\right)=\mathfrak{K}_{1}$; hence, the site $\mathfrak{y}_{1}$ is decided to be the most suitable site for the opening of a new cafe outlet.

To better understand Algorithm 1, its flowchart diagram is shown in Figure 2.

Now, we present the next application of $\mathrm{FP}^{q}$ ROFSESs.

3.2. Selection of Best News Channel. News channels and journalists all over the world keep us updated about the events in the world, the changes in policies, the crisis coverage, and a lot more. The main purposes, including informing, guiding, educating, and entertaining the audience, interpreting the news and facts, causing awareness for social issues, and forming value opinions about the policies and situations, etc., make these news channels and journalism a very important part of the society. With the advancements in technology, many changes have come to journalism too. First, we were informed with news in black and white and then came the radio tech and then the TV networks, and now, the online services allow the availability of news in any place at any instant.

Although all the news channels try their best to keep their audience best updated and entertained, many factors like biased opinions, forced news, low quality, lack of factual material, and so on, can affect the quality of a news channel to a huge extent. For this, every year, many organizations (like AVTA, RTS, CNA, and CRN) award the channels by critical analysis of the channels in their respective domains, which helps to find the credibility of a news channel effectively. In this example, we model a problem where an award of "Best News Channel" is to be awarded on the basis of various criteria.

Consider that an organization announces an event for the award of "Best News Channel," for which eight highrated news channels are nominated. The nominated channels are presented in the set $\mathscr{Y}=\left\{\mathfrak{y}_{1}, \mathfrak{y}_{2}, \ldots, \mathfrak{y}_{8}\right\}$. For the analysis of the channels, a committee of three experts as in the set $\mathscr{E}=\left\{\boldsymbol{e}_{1}, \boldsymbol{e}_{2}, \boldsymbol{e}_{3}\right\}$ is considered by the organization. These experts choose a favorable set of parameters given as $\mathcal{S}=\left\{\mathfrak{G}_{1}, \mathfrak{G}_{2}, \mathfrak{G}_{3}, \mathfrak{S}_{4}, \mathfrak{G}_{5}\right\}$ for the judgments, where $\mathfrak{S}_{i}(i=1,2,3,4,5)$ stand for "effective social awareness," "quality entertainment," "unbiased quality opinions," "factual information," and "quality guidance and education." The weights assigned to the parameters by the experts are $\mathscr{D}=\left\{\left(0.6 / \mathfrak{g}_{1}\right),\left(0.2 / \mathfrak{G}_{2}\right),\left(0.9 / \mathfrak{g}_{3}\right),\left(0.7 / \mathfrak{g}_{4}\right),\left(0.3 / \mathfrak{G}_{5}\right)\right\}$. After critically analyzing the channels, the committee provides the data, which is represented by an $\operatorname{FP}^{q} \operatorname{ROFSES}(\mathfrak{f}, \mathscr{Z})_{\mathscr{D}}$ with $q=5$ as follows:

$$
\begin{aligned}
(\mathfrak{f}, \mathscr{X})_{\mathscr{D}}= & \left\langle\left(\frac{0.6}{\mathfrak{g}_{1}}, \mathfrak{e}_{1}, 1\right)\left\{\frac{\mathfrak{y}_{1}}{(0.79,0.91)}, \frac{\mathfrak{y}_{2}}{(0.58,0.26)}, \frac{\mathfrak{y}_{3}}{(0.53,0.48)}, \frac{\mathfrak{y}_{4}}{(0.85,0.23)}, \frac{\mathfrak{y}_{5}}{(0.19,0.37)}, \frac{\mathfrak{y}_{6}}{(0.49,0.39)}, \frac{\mathfrak{y}_{7}}{(0.62,0.87)}, \frac{\mathfrak{y}_{8}}{(0.13,0.86)}\right\}\right\rangle, \\
& \left\langle\left(\frac{0.2}{\mathfrak{g}_{2}}, \mathfrak{e}_{1}, 1\right)\left\{\frac{\mathfrak{y}_{1}}{(0.17,0.28)}, \frac{\mathfrak{y}_{2}}{(0.96,0.36)}, \frac{\mathfrak{y}_{3}}{(0.17,0.48)}, \frac{\mathfrak{y}_{4}}{(0.89,0.13)}, \frac{\mathfrak{y}_{5}}{(0.25,0.28)}, \frac{\mathfrak{y}_{6}}{(0.59,0.49)}, \frac{\mathfrak{y}_{7}}{(0.81,0.75)}, \frac{\mathfrak{y}_{8}}{(0.34,0.13)}\right\}\right\rangle, \\
& \left\langle\left(\frac{0.9}{\mathfrak{g}_{3}}, \mathfrak{e}_{1}, 1\right)\left\{\frac{\mathfrak{y}_{1}}{(0.25,0.61)}, \frac{\mathfrak{y}_{2}}{(0.64,0.88)}, \frac{\mathfrak{y}_{3}}{(0.85,0.67)}, \frac{\mathfrak{y}_{4}}{(0.49,0.20)}, \frac{\mathfrak{y}_{5}}{(0.41,0.35)}, \frac{\mathfrak{y}_{6}}{(0.79,0.69)}, \frac{\mathfrak{y}_{7}}{(0.65,0.24)}, \frac{\mathfrak{y}_{8}}{(0.57,0.12)}\right\}\right\rangle, \\
& \left\langle\left(\frac{0.7}{\mathfrak{g}_{4}}, \mathfrak{e}_{1}, 1\right)\left\{\frac{\mathfrak{y}_{1}}{(0.32,0.31)}, \frac{\mathfrak{y}_{2}}{(0.73,0.55)}, \frac{\mathfrak{y}_{3}}{(0.60,0.75)}, \frac{\mathfrak{y}_{4}}{(0.26,0.56)}, \frac{\mathfrak{y}_{5}}{(0.19,0.90)}, \frac{\mathfrak{y}_{6}}{(0.28,0.16)}, \frac{\mathfrak{y}_{7}}{(0.31,0.54)}, \frac{\mathfrak{y}}{(0.39,0.23)}\right\}\right\rangle,
\end{aligned}
$$


(1) Input

$\mathcal{Y}$, a universal set,

$\mathcal{S}$, a universe of parameters,

$\mathscr{E}$, a set of experts,

$\mathscr{D}$, a set of weights for parameters,

$\mathcal{O}$, a set of opinions,

$(\mathfrak{f}, \mathscr{Z})_{\mathscr{D}}$, an $\mathrm{FP}^{q}$ ROFSES.

(2) Find the table of scores with entries $X_{i j}$ by Definition 14.

(3) Find the table of scores for agree-FP ${ }^{q}$ ROFSES.

(4) Find the table of scores for disagree-FP $q$ ROFSES.

(5) Input the accumulated agree scores $L_{j}=\sum_{i} w_{i} X_{i j}$ in the last row of the score table of agree-FP $\mathrm{ROFSES}^{q}$

(6) Input the accumulated disagree scores $C_{j}=\sum_{i} w_{i} X_{i j}$ in the last row of the score table of disagree-FP ${ }^{q}$ ROFSES.

(7) Find the values of final scores $\mathfrak{\Re}_{j}=L_{j}-C_{j}$.

(8) Find $m$, for which $\mathfrak{\Re}_{m}=\max \mathfrak{\Re}_{j}$.

(9) Output

In step (8), the object $\mathfrak{y}_{m}$ having maximum final score will be the most suitable choice. If there exist two or more objects with maximum final scores, then anyone can be chosen as a decision.

Algorithm 1: Decision-making method based on FP ${ }^{q}$ ROFSESs.

TAble 1: Score table for $\mathrm{FP}^{4}$ ROFSES $(\mathfrak{f}, \mathscr{Z})_{\mathscr{D}}$.

\begin{tabular}{|c|c|c|c|c|c|}
\hline$y$ & $\mathfrak{y}_{1}$ & $\mathfrak{y}_{2}$ & $\mathfrak{y}_{3}$ & $\mathfrak{y}_{4}$ & $\mathfrak{y}_{5}$ \\
\hline$\left(\left(0.5 / \mathfrak{G}_{1}\right), \mathfrak{e}_{1}, 1\right)$ & 0.4160 & 0.0103 & -0.1383 & 0.1695 & 0.0644 \\
\hline$\left(\left(0.3 / \mathfrak{g}_{2}\right), \mathfrak{e}_{1}, 1\right)$ & -0.0198 & -0.0048 & 0.0335 & 0.0602 & 0.2099 \\
\hline$\left(\left(0.8 / \mathfrak{G}_{3}\right), \mathfrak{e}_{1}, 1\right)$ & -0.0117 & 0.3873 & 0.4730 & -0.0035 & -0.2933 \\
\hline$\left(\left(0.5 / \mathfrak{g}_{1}\right), \mathrm{e}_{2}, 1\right)$ & -0.2488 & -0.1375 & -0.3699 & -0.1704 & 0.6219 \\
\hline$\left(\left(0.3 / \mathfrak{G}_{2}\right), \mathfrak{e}_{2}, 1\right)$ & -0.0357 & -0.0034 & -0.0014 & 0.2262 & 0.0131 \\
\hline$\left(\left(0.8 / \mathfrak{G}_{3}\right), \mathfrak{e}_{2}, 1\right)$ & -0.3930 & -0.1375 & -0.0178 & 0.0043 & 0.5863 \\
\hline$\left(\left(0.5 / \mathfrak{g}_{1}\right), \mathfrak{e}_{3}, 1\right)$ & -0.0990 & -0.0029 & 0.0088 & -0.0250 & 0.0441 \\
\hline$\left(\left(0.3 / \mathfrak{G}_{2}\right), \mathfrak{e}_{3}, 1\right)$ & -0.2800 & -0.0455 & 0.1024 & -0.0052 & 0.1662 \\
\hline$\left(\left(0.8 / \mathfrak{G}_{3}\right), \mathfrak{e}_{3}, 1\right)$ & 0.3574 & 0.0536 & -0.1762 & 0.7030 & -0.0796 \\
\hline$\left(\left(0.5 / \mathfrak{g}_{1}\right), \mathfrak{e}_{4}, 1\right)$ & 0.2313 & -0.0802 & 0.1407 & -0.0335 & -0.4015 \\
\hline$\left(\left(0.3 / \mathfrak{G}_{2}\right), \mathfrak{e}_{4}, 1\right)$ & 0.1730 & -0.2313 & -0.0200 & -0.0573 & 0.0365 \\
\hline$\left(\left(0.8 / \mathfrak{G}_{3}\right), \mathfrak{e}_{4}, 1\right)$ & 0.5319 & 0.1166 & 0.1940 & 0.0077 & 0.2382 \\
\hline$\left(\left(0.5 / \mathfrak{g}_{1}\right), \mathfrak{e}_{4}, 0\right)$ & 0.3270 & 0.4252 & 0.4354 & 0.5214 & -0.0018 \\
\hline$\left(\left(0.3 / \mathfrak{g}_{2}\right), \mathfrak{e}_{1}, 0\right)$ & -0.0018 & -0.0031 & -0.0010 & -0.0011 & -0.0032 \\
\hline$\left(\left(0.8 / \mathfrak{g}_{3}\right), \mathfrak{e}_{1}, 0\right)$ & -0.0219 & -0.0031 & -0.0027 & -0.0022 & -0.0314 \\
\hline$\left(\left(0.5 / \mathfrak{g}_{1}\right), \mathfrak{e}_{2}, 0\right)$ & -0.0044 & -0.2391 & -0.0036 & -0.0202 & -0.0260 \\
\hline$\left(\left(0.3 / \mathfrak{g}_{2}\right), \mathfrak{e}_{2}, 0\right)$ & -0.0122 & 0.0161 & 0.0155 & -0.0011 & 0.0509 \\
\hline$\left(\left(0.8 / \mathfrak{G}_{3}\right), \mathfrak{e}_{2}, 0\right)$ & 0.0827 & -0.0185 & 0.0910 & 0.1477 & 0.0404 \\
\hline$\left(\left(0.5 / \mathfrak{s}_{1}\right), \mathfrak{e}_{3}, 0\right)$ & 0.1165 & 0.0230 & 0.1665 & 0.1570 & 0.1469 \\
\hline$\left(\left(0.3 / \mathfrak{G}_{2}\right), \mathfrak{e}_{3}, 0\right)$ & 0.2247 & 0.2533 & 0.2583 & 0.2801 & -0.0079 \\
\hline$\left(\left(0.8 / \mathfrak{g}_{3}\right), \mathfrak{e}_{3}, 0\right)$ & 0.0088 & 0.0043 & -0.0059 & -0.1375 & -0.0747 \\
\hline$\left(\left(0.5 / \mathfrak{G}_{1}\right), \mathfrak{e}_{4}, 0\right)$ & -0.0117 & 0.1141 & -0.0289 & -0.0990 & 0.3574 \\
\hline$\left(\left(0.3 / \mathfrak{G}_{2}\right), \mathfrak{e}_{4}, 0\right)$ & 0.0335 & 0.2345 & -0.0034 & 0.0088 & 0.1024 \\
\hline$\left(\left(0.8 / \mathfrak{g}_{3}\right), \mathfrak{e}_{4}, 0\right)$ & -0.0178 & -0.0455 & 0.0014 & 0.4978 & 0.7030 \\
\hline
\end{tabular}


TABLe 2: Accumulated score table for agree-FP ${ }^{4} \operatorname{ROFSES}(\mathfrak{f}, \mathscr{Z})_{\mathscr{D}}^{1}$.

\begin{tabular}{|c|c|c|c|c|c|}
\hline$y$ & $\mathfrak{y}_{1}$ & $\mathfrak{y}_{2}$ & $\mathfrak{y}_{3}$ & $\mathfrak{y}_{4}$ & $\mathfrak{y}_{5}$ \\
\hline$\left(\left(0.5 / \mathfrak{g}_{1}\right), \mathfrak{e}_{1}, 1\right)$ & 0.4160 & 0.0103 & -0.1383 & 0.1695 & 0.0644 \\
\hline$\left(\left(0.3 / \mathfrak{s}_{2}\right), \mathfrak{e}_{1}, 1\right)$ & -0.0198 & -0.0048 & 0.0335 & 0.0602 & 0.2099 \\
\hline$\left(\left(0.8 / \mathfrak{G}_{3}\right), \mathfrak{e}_{1}, 1\right)$ & -0.0117 & 0.3873 & 0.4730 & -0.0035 & -0.2933 \\
\hline$\left(\left(0.5 / \mathfrak{s}_{1}\right), \mathfrak{e}_{2}, 1\right)$ & -0.2488 & -0.1375 & -0.3699 & -0.1704 & 0.6219 \\
\hline$\left(\left(0.3 / \mathfrak{g}_{2}\right), \mathfrak{e}_{2}, 1\right)$ & -0.0357 & -0.0034 & -0.0014 & 0.2262 & 0.0131 \\
\hline$\left(\left(0.8 / \mathfrak{s}_{3}\right), \mathfrak{e}_{2}, 1\right)$ & -0.3930 & -0.1375 & -0.0178 & 0.0043 & 0.5863 \\
\hline$\left(\left(0.5 / \mathfrak{g}_{1}\right), \mathfrak{e}_{3}, 1\right)$ & -0.0990 & -0.0029 & 0.0088 & -0.0250 & 0.0441 \\
\hline$\left(\left(0.3 / \mathfrak{g}_{2}\right), \mathfrak{e}_{3}, 1\right)$ & -0.2800 & -0.0455 & 0.1024 & -0.0052 & 0.1662 \\
\hline$\left(\left(0.8 / \mathfrak{s}_{3}\right), \mathfrak{e}_{3}, 1\right)$ & 0.3574 & 0.0536 & -0.1762 & 0.7030 & -0.0796 \\
\hline$\left(\left(0.5 / \mathfrak{g}_{1}\right), \mathfrak{e}_{4}, 1\right)$ & 0.2313 & -0.0802 & 0.1407 & -0.0335 & -0.4015 \\
\hline$\left(\left(0.3 / \mathfrak{g}_{2}\right), \mathfrak{e}_{4}, 1\right)$ & 0.1730 & -0.2313 & -0.0200 & -0.0573 & 0.0365 \\
\hline$\left(\left(0.8 / \mathfrak{G}_{3}\right), \mathfrak{e}_{4}, 1\right)$ & 0.5319 & 0.1166 & 0.1940 & 0.0077 & 0.2382 \\
\hline$L_{j}=\sum_{i} w_{i} X_{i j}$ & $L_{1}=0.4887$ & $L_{2}=0.1454$ & $L_{3}=0.2334$ & $L_{4}=0.6067$ & $L_{5}=0.6534$ \\
\hline
\end{tabular}

TABLE 3: Accumulated score table for disagree-FP ${ }^{4}$ ROFSES $(\mathfrak{f}, \mathscr{Z})_{\mathscr{D}}^{0}$.

\begin{tabular}{|c|c|c|c|c|c|}
\hline$y$ & $\mathfrak{y}_{1}$ & $\mathfrak{y}_{2}$ & $\mathfrak{y}_{3}$ & $\mathfrak{y}_{4}$ & $\mathfrak{y}_{5}$ \\
\hline$\left(\left(0.5 / \mathfrak{G}_{1}\right), \mathfrak{e}_{1}, 0\right)$ & 0.3270 & 0.4252 & 0.4354 & 0.5214 & -0.0018 \\
\hline$\left(\left(0.3 / \mathfrak{G}_{2}\right), \mathfrak{e}_{1}, 0\right)$ & -0.0018 & -0.0031 & -0.0010 & -0.0011 & -0.0032 \\
\hline$\left(\left(0.8 / \mathfrak{G}_{3}\right), \mathfrak{e}_{1}, 0\right)$ & -0.0219 & -0.0031 & -0.0027 & -0.0022 & -0.0314 \\
\hline$\left(\left(0.5 / \mathfrak{s}_{1}\right), \mathfrak{e}_{2}, 0\right)$ & -0.0044 & -0.2391 & -0.0036 & -0.0202 & -0.0260 \\
\hline$\left(\left(0.3 / \mathfrak{G}_{2}\right), \mathfrak{e}_{2}, 0\right)$ & -0.0122 & 0.0161 & 0.0155 & -0.0011 & 0.0509 \\
\hline$\left(\left(0.8 / \mathfrak{G}_{3}\right), \mathfrak{e}_{2}, 0\right)$ & 0.0827 & -0.0185 & 0.0910 & 0.1477 & 0.0404 \\
\hline$\left(\left(0.5 / \mathfrak{G}_{1}\right), \mathfrak{e}_{3}, 0\right)$ & 0.1165 & 0.0230 & 0.1665 & 0.1570 & 0.1469 \\
\hline$\left(\left(0.3 / \mathfrak{g}_{2}\right), \mathfrak{e}_{3}, 0\right)$ & 0.2247 & 0.2533 & 0.2583 & 0.2801 & -0.0079 \\
\hline$\left(\left(0.8 / \mathfrak{G}_{3}\right), \mathfrak{e}_{3}, 0\right)$ & 0.0088 & 0.0043 & -0.0059 & -0.1375 & -0.0747 \\
\hline$\left(\left(0.5 / \mathfrak{G}_{1}\right), \mathfrak{e}_{4}, 0\right)$ & -0.0117 & 0.1141 & -0.0289 & -0.0990 & 0.3574 \\
\hline$\left(\left(0.3 / \mathfrak{G}_{2}\right), \mathfrak{e}_{4}, 0\right)$ & 0.0335 & 0.2345 & -0.0034 & 0.0088 & 0.1024 \\
\hline$\left(\left(0.8 / \mathfrak{G}_{3}\right), \mathfrak{e}_{4}, 0\right)$ & -0.0178 & -0.0455 & 0.0014 & 0.4978 & 0.7030 \\
\hline$C_{j}=\sum_{i} w_{i} X_{i j}$ & $C_{1}=0.3285$ & $C_{2}=0.2616$ & $C_{3}=0.4325$ & $C_{4}=0.7703$ & $C_{5}=0.7907$ \\
\hline
\end{tabular}

TABle 4: Final scores.

\begin{tabular}{|c|c|c|}
\hline$L_{j}$ & $C_{j}$ & $\mathfrak{\Re}_{j}=L_{j}-C_{j}$ \\
\hline$L_{1}=0.4887$ & $C_{1}=0.3285$ & $\mathfrak{K}_{1}=0.1603$ \\
\hline$L_{2}=0.1454$ & $C_{2}=0.2616$ & $\mathfrak{K}_{2}=-0.1162$ \\
\hline$L_{3}=0.2334$ & $C_{3}=0.4325$ & $\mathfrak{K}_{3}=-0.1991$ \\
\hline$L_{4}=0.6067$ & $C_{4}=0.7703$ & $\mathfrak{\mathfrak { R }}_{4}=-0.1636$ \\
\hline$L_{5}=0.6534$ & $C_{5}=0.7909$ & $\mathfrak{\mathfrak { N }}_{5}=-0.1373$ \\
\hline
\end{tabular}



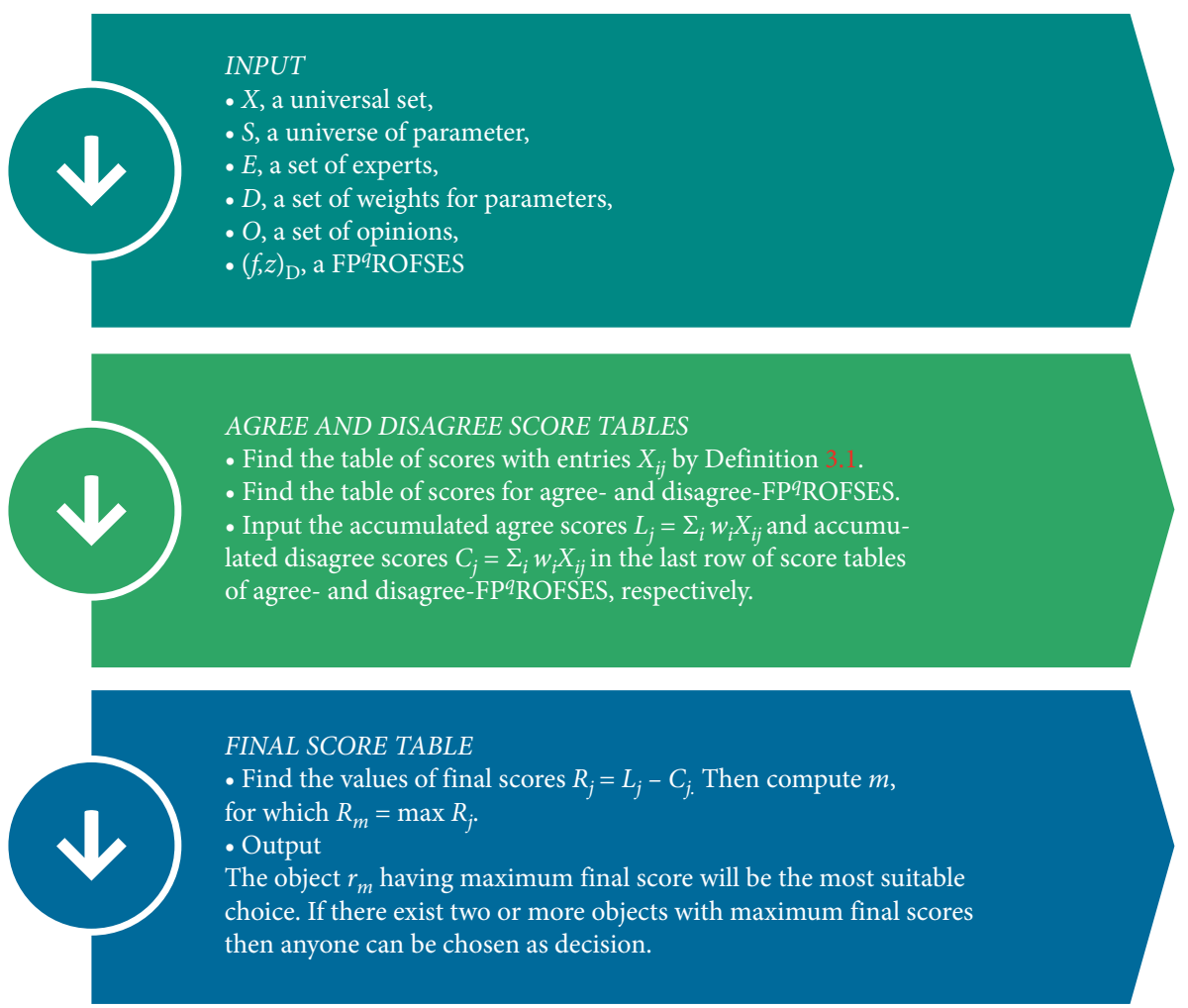

Figure 2: Flowchart diagram.

TAble 5: Score table for $\mathrm{FP}^{5}$ ROFSES $(\mathfrak{f}, \mathscr{Z})_{\mathscr{D}}$.

\begin{tabular}{|c|c|c|c|c|c|c|c|c|}
\hline$y$ & $\mathfrak{y}_{1}$ & $\mathfrak{y}_{2}$ & $\mathfrak{y}_{3}$ & $\mathfrak{y}_{4}$ & $\mathfrak{y}_{5}$ & $\mathfrak{y}_{6}$ & $\mathfrak{y}_{7}$ & $\mathfrak{y}_{8}$ \\
\hline$\left(\left(0.6 / \mathfrak{g}_{1}\right), \mathfrak{e}_{1}, 1\right)$ & -0.3163 & 0.0644 & 0.0163 & 0.4431 & -0.0067 & 0.0192 & -0.2571 & -0.4704 \\
\hline$\left(\left(0.2 / \mathfrak{g}_{2}\right), \mathfrak{e}_{1}, 1\right)$ & -0.0016 & 0.8093 & -0.0253 & 0.5584 & -0.0007 & 0.0432 & 0.1114 & 0.0045 \\
\hline$\left(\left(0.9 / \mathfrak{G}_{3}\right), \mathfrak{e}_{1}, 1\right)$ & -0.0835 & -0.4204 & 0.3087 & 0.0279 & 0.0063 & 0.1513 & 0.1152 & 0.0601 \\
\hline$\left(\left(0.7 / \mathfrak{g}_{4}\right), \mathfrak{e}_{1}, 1\right)$ & 0.0005 & 0.1570 & -0.1595 & -0.0539 & -0.5902 & 0.0016 & -0.0431 & 0.0084 \\
\hline$\left(\left(0.3 / \mathfrak{g}_{5}\right), \mathfrak{e}_{1}, 1\right)$ & 0.0345 & 0.5515 & 0.0041 & -0.1720 & 0.0070 & -0.2203 & 0.0085 & 0.0019 \\
\hline$\left(\left(0.6 / \mathfrak{g}_{1}\right), \mathfrak{e}_{2}, 1\right)$ & -0.1368 & 0.0082 & -0.0049 & -0.2028 & -0.0133 & 0.0045 & 0.2499 & -0.0687 \\
\hline$\left(\left(0.2 / \mathfrak{g}_{2}\right), \mathfrak{e}_{2}, 1\right)$ & 0.0051 & -0.0015 & 0.4806 & 0.0976 & 0.0010 & 0.3322 & 0.0012 & 0.0221 \\
\hline$\left(\left(0.9 / \mathfrak{g}_{3}\right), \mathfrak{e}_{2}, 1\right)$ & 0.0844 & 0.0146 & 0.3925 & 0.2887 & -0.0005 & 0.0014 & 0.3342 & -0.0014 \\
\hline$\left(\left(0.7 / \mathfrak{G}_{4}\right), \mathfrak{e}_{2}, 1\right)$ & -0.5803 & 0.0427 & 0.1504 & -0.2283 & 0.5758 & 0.0714 & -0.0668 & 0.0205 \\
\hline$\left(\left(0.3 / \mathfrak{g}_{5}\right), \mathfrak{e}_{2}, 1\right)$ & 0.1071 & -0.0481 & 0.3107 & 0.0345 & -0.1155 & 0.7294 & -0.0012 & -0.0010 \\
\hline$\left(\left(0.6 / \mathfrak{s}_{1}\right), \mathfrak{e}_{3}, 1\right)$ & -0.0701 & -0.0198 & 0.1770 & 0.0087 & 0.0210 & 0.3017 & -0.0104 & 0.1192 \\
\hline$\left(\left(0.2 / \mathfrak{g}_{2}\right), \mathfrak{e}_{3}, 1\right)$ & 0.0312 & 0.2964 & 0.5127 & 0.1677 & 0.3277 & 0.0021 & 0.0309 & -0.1578 \\
\hline$\left(\left(0.9 / \mathfrak{g}_{3}\right), \mathfrak{e}_{3}, 1\right)$ & 0.0003 & 0.1804 & -0.0116 & 0.3483 & 0.1771 & 0.2642 & 0.3276 & 0.0102 \\
\hline$\left(\left(0.7 / \mathfrak{g}_{4}\right), \mathfrak{e}_{3}, 1\right)$ & -0.1770 & 0.4800 & 0.0785 & -0.0536 & 0.1714 & 0.0610 & 0.3253 & 0.0175 \\
\hline$\left(\left(0.3 / \mathfrak{g}_{5}\right), \mathfrak{e}_{3}, 1\right)$ & -0.0014 & 0.0051 & 0.0932 & -0.1683 & 0.0426 & 0.0214 & 0.0366 & 0.0125 \\
\hline$\left(\left(0.6 / \mathfrak{g}_{1}\right), \mathfrak{e}_{1}, 0\right)$ & 0.0024 & 0.5904 & 0.1596 & 0.1578 & 0.3253 & 0.0312 & 0.0844 & 0.0753 \\
\hline$\left(\left(0.2 / \mathfrak{g}_{2}\right), \mathfrak{e}_{1}, 0\right)$ & 0.5437 & 0.1525 & -0.6498 & -0.0584 & 0.2373 & 0.1681 & 0.1268 & 0.7337 \\
\hline$\left(\left(0.9 / \mathfrak{G}_{3}\right), \mathfrak{e}_{1}, 0\right)$ & 0.2964 & 0.0001 & 0.0003 & 0.0260 & 0.0144 & -0.1222 & 0.1823 & 0.2765 \\
\hline$\left(\left(0.7 / \mathfrak{g}_{4}\right), \mathfrak{e}_{1}, 0\right)$ & 0.0004 & 0.0008 & 0.0020 & 0.0005 & 0.0021 & 0.0132 & 0.0065 & 0.0454 \\
\hline$\left(\left(0.7 / \mathfrak{G}_{5}\right), \mathfrak{e}_{1}, 0\right)$ & 0.1578 & 0.0465 & -0.0768 & 0.0708 & 0.1516 & -0.5303 & 0.1833 & 0.3484 \\
\hline$\left(\left(0.3 / \mathfrak{G}_{1}\right), \mathfrak{e}_{2}, 0\right)$ & 0.0008 & 0.0028 & 0.0102 & 0.0161 & 0.1679 & 0.0057 & 0.0002 & 0.0028 \\
\hline$\left(\left(0.6 / \mathfrak{g}_{2}\right), \mathfrak{e}_{2}, 0\right)$ & 0.5881 & 0.0024 & 0.0028 & 0.1601 & 0.0226 & 0.0841 & 0.1595 & 0.3870 \\
\hline$\left(\left(0.2 / \mathfrak{G}_{3}\right), \mathfrak{e}_{2}, 0\right)$ & 0.0012 & 0.0011 & 0.0008 & 0.0008 & 0.5901 & -0.0009 & 0.0070 & 0.0266 \\
\hline$\left(\left(0.9 / \mathfrak{g}_{4}\right), \mathfrak{e}_{2}, 0\right)$ & 0.0309 & 0.0005 & 0.0001 & 0.0184 & 0.0243 & 0.2413 & 0.1634 & 0.4588 \\
\hline$\left(\left(0.7 / \mathfrak{G}_{5}\right), \mathfrak{e}_{2}, 0\right)$ & 0.0003 & 0.0009 & -0.0005 & -0.1677 & 0.0105 & 0.0156 & 0.0001 & 0.3472 \\
\hline$\left(\left(0.3 / \mathfrak{g}_{1}\right), \mathfrak{e}_{3}, 0\right)$ & 0.0916 & 0.0144 & 0.0184 & 0.1071 & 0.1560 & 0.1803 & 0.0992 & 0.0808 \\
\hline$\left(\left(0.6 / \mathfrak{g}_{2}\right), \mathfrak{e}_{3}, 0\right)$ & 0.0844 & 0.0183 & 0.0028 & 0.0503 & 0.0045 & 0.0045 & 0.0089 & 0.0915 \\
\hline$\left(\left(0.2 / \mathfrak{g}_{3}\right), \mathfrak{e}_{3}, 0\right)$ & 0.1901 & 0.2063 & 0.1368 & 0.0015 & 0.3936 & 0.0517 & 0.0714 & 0.2842 \\
\hline$\left(\left(0.9 / \mathfrak{g}_{4}\right), \mathfrak{e}_{3}, 0\right)$ & 0.0082 & 0.4806 & -0.2887 & 0.0005 & 0.0014 & 0.0205 & 0.1071 & -0.0481 \\
\hline$\left(\left(0.7 / \mathfrak{g}_{5}\right), \mathfrak{e}_{3}, 0\right)$ & 0.0427 & 0.0349 & 0.3936 & 0.0976 & 0.0133 & -0.0045 & -0.0505 & 0.3107 \\
\hline
\end{tabular}


TABLE 6: Accumulated score table for agree-FP ${ }^{5}$ ROFSES $(\mathfrak{f}, \mathscr{Z})_{\mathscr{D}}^{1}$.

\begin{tabular}{|c|c|c|c|c|c|c|c|c|}
\hline $\mathscr{y}$ & $\mathfrak{y}_{1}$ & $\mathfrak{y}_{2}$ & $\mathfrak{y}_{3}$ & $\mathfrak{y}_{4}$ & $\mathfrak{y}_{5}$ & $\mathfrak{y}_{6}$ & $\mathfrak{y}_{7}$ & $\mathfrak{y}_{8}$ \\
\hline$\left(\left(0.6 / \mathfrak{s}_{1}\right), \mathfrak{e}_{1}, 1\right)$ & -0.3163 & 0.0644 & 0.0163 & 0.4431 & -0.0067 & 0.0192 & -0.2571 & -0.4704 \\
\hline$\left(\left(0.2 / \mathfrak{g}_{2}\right), \mathfrak{e}_{1}, 1\right)$ & -0.0016 & 0.8093 & -0.0253 & 0.5584 & -0.0007 & 0.0432 & 0.1114 & 0.0045 \\
\hline$\left(\left(0.9 / \mathfrak{s}_{3}\right), \mathfrak{e}_{1}, 1\right)$ & -0.0835 & -0.4204 & 0.3087 & 0.0279 & 0.0063 & 0.1513 & 0.1152 & 0.0601 \\
\hline$\left(\left(0.7 / \mathfrak{g}_{4}\right), \mathfrak{e}_{1}, 1\right)$ & 0.0005 & 0.1570 & -0.1595 & -0.0539 & -0.5902 & 0.0016 & -0.0431 & 0.0084 \\
\hline$\left(\left(0.3 / \mathfrak{g}_{5}\right), \mathfrak{e}_{1}, 1\right)$ & 0.0345 & 0.5515 & 0.0041 & -0.1720 & 0.0070 & -0.2203 & 0.0085 & 0.0019 \\
\hline$\left(\left(0.6 / \mathfrak{G}_{1}\right), \mathfrak{e}_{2}, 1\right)$ & -0.1368 & 0.0082 & -0.0049 & -0.2028 & -0.0133 & 0.0045 & 0.2499 & -0.0687 \\
\hline$\left(\left(0.2 / \mathfrak{g}_{2}\right), \mathfrak{e}_{2}, 1\right)$ & 0.0051 & -0.0015 & 0.4806 & 0.0976 & 0.0010 & 0.3322 & 0.0012 & 0.0221 \\
\hline$\left(\left(0.9 / \mathfrak{g}_{3}\right), \mathfrak{e}_{2}, 1\right)$ & 0.0844 & 0.0146 & 0.3925 & 0.2887 & -0.0005 & 0.0014 & 0.3342 & -0.0014 \\
\hline$\left(\left(0.7 / \mathfrak{s}_{4}\right), \mathfrak{e}_{2}, 1\right)$ & -0.5803 & 0.0427 & 0.1504 & -0.2283 & 0.5758 & 0.0714 & -0.0668 & 0.0205 \\
\hline$\left(\left(0.3 / \mathfrak{g}_{5}\right), \mathfrak{e}_{2}, 1\right)$ & 0.1071 & -0.0481 & 0.3107 & 0.0345 & -0.1155 & 0.7294 & -0.0012 & -0.0010 \\
\hline$\left(\left(0.6 / \mathfrak{s}_{1}\right), \mathfrak{e}_{3}, 1\right)$ & -0.0701 & -0.0198 & 0.1770 & 0.0087 & 0.0210 & 0.3017 & -0.0104 & 0.1192 \\
\hline$\left(\left(0.2 / \mathfrak{g}_{2}\right), \mathfrak{e}_{3}, 1\right)$ & 0.0312 & 0.2964 & 0.5127 & 0.1677 & 0.3277 & 0.0021 & 0.0309 & -0.1578 \\
\hline$\left(\left(0.9 / \mathfrak{s}_{3}\right), \mathfrak{e}_{3}, 1\right)$ & 0.0003 & 0.1804 & -0.0116 & 0.3483 & 0.1771 & 0.2642 & 0.3276 & 0.0102 \\
\hline$\left(\left(0.7 / \mathfrak{G}_{4}\right), \mathfrak{e}_{3}, 1\right)$ & -0.1770 & 0.4800 & 0.0785 & -0.0536 & 0.1714 & 0.0610 & 0.3253 & 0.0175 \\
\hline$\left(\left(0.3 / \mathfrak{g}_{5}\right), \mathfrak{e}_{3}, 1\right)$ & -0.0014 & 0.0051 & 0.0932 & -0.1683 & 0.0426 & 0.0214 & 0.0366 & 0.0125 \\
\hline$L_{j}=\sum_{i} w_{i} X_{i j}$ & -0.7936 & 0.6780 & 1.0982 & 0.5857 & 0.3210 & 0.8989 & 0.8814 & -0.1797 \\
\hline
\end{tabular}

TABLE 7: Accumulated score table for disagree-FP ${ }^{5} \operatorname{ROFSES}(\mathfrak{f}, \mathscr{Z})_{\mathscr{D}}^{0}$.

\begin{tabular}{|c|c|c|c|c|c|c|c|c|}
\hline$y$ & $\mathfrak{y}_{1}$ & $\mathfrak{y}_{2}$ & $\mathfrak{y}_{3}$ & $\mathfrak{y}_{4}$ & $\mathfrak{y}_{5}$ & $\mathfrak{y}_{6}$ & $\mathfrak{y}_{7}$ & $\mathfrak{y}_{8}$ \\
\hline$\left(\left(0.6 / \mathfrak{g}_{1}\right), \mathfrak{e}_{1}, 0\right)$ & 0.0024 & 0.5904 & 0.1596 & 0.1578 & 0.3253 & 0.0312 & 0.0844 & 0.0753 \\
\hline$\left(\left(0.2 / \mathfrak{G}_{2}\right), \mathfrak{e}_{1}, 0\right)$ & 0.5437 & 0.1525 & -0.6498 & -0.0584 & 0.2373 & 0.1681 & 0.1268 & 0.7337 \\
\hline$\left(\left(0.9 / \mathfrak{g}_{3}\right), \mathfrak{e}_{1}, 0\right)$ & 0.2964 & 0.0001 & 0.0003 & 0.0260 & 0.0144 & -0.1222 & 0.1823 & 0.2765 \\
\hline$\left(\left(0.7 / \mathfrak{G}_{4}\right), \mathfrak{e}_{1}, 0\right)$ & 0.0004 & 0.0008 & 0.0020 & 0.0005 & 0.0021 & 0.0132 & 0.0065 & 0.0454 \\
\hline$\left(\left(0.7 / \mathfrak{g}_{5}\right), \mathfrak{e}_{1}, 0\right)$ & 0.1578 & 0.0465 & -0.0768 & 0.0708 & 0.1516 & -0.5303 & 0.1833 & 0.3484 \\
\hline$\left(\left(0.3 / \mathfrak{g}_{1}\right), \mathfrak{e}_{2}, 0\right)$ & 0.0008 & 0.0028 & 0.0102 & 0.0161 & 0.1679 & 0.0057 & 0.0002 & 0.0028 \\
\hline$\left(\left(0.6 / \mathfrak{g}_{2}\right), \mathfrak{e}_{2}, 0\right)$ & 0.5881 & 0.0024 & 0.0028 & 0.1601 & 0.0226 & 0.0841 & 0.1595 & 0.3870 \\
\hline$\left(\left(0.2 / \mathfrak{G}_{3}\right), \mathfrak{e}_{2}, 0\right)$ & 0.0012 & 0.0011 & 0.0008 & 0.0008 & 0.5901 & -0.0009 & 0.0070 & 0.0266 \\
\hline$\left(\left(0.9 / \mathfrak{g}_{4}\right), \mathfrak{e}_{2}, 0\right)$ & 0.0309 & 0.0005 & 0.0001 & 0.0184 & 0.0243 & 0.2413 & 0.1634 & 0.4588 \\
\hline$\left(\left(0.7 / \mathfrak{g}_{5}\right), \mathfrak{e}_{2}, 0\right)$ & 0.0003 & 0.0009 & -0.0005 & -0.1677 & 0.0105 & 0.0156 & 0.0001 & 0.3472 \\
\hline$\left(\left(0.3 / \mathfrak{s}_{1}\right), \mathfrak{e}_{3}, 0\right)$ & 0.0916 & 0.0144 & 0.0184 & 0.1071 & 0.1560 & 0.1803 & 0.0992 & 0.0808 \\
\hline$\left(\left(0.6 / \mathfrak{G}_{2}\right), \mathfrak{e}_{3}, 0\right)$ & 0.0844 & 0.0183 & 0.0028 & 0.0503 & 0.0045 & 0.0045 & 0.0089 & 0.0915 \\
\hline$\left(\left(0.2 / \mathfrak{g}_{3}\right), \mathfrak{e}_{3}, 0\right)$ & 0.1901 & 0.2063 & 0.1368 & 0.0015 & 0.3936 & 0.0517 & 0.0714 & 0.2842 \\
\hline$\left(\left(0.9 / \mathfrak{g}_{4}\right), \mathfrak{e}_{3}, 0\right)$ & 0.0082 & 0.4806 & -0.2887 & 0.0005 & 0.0014 & 0.0205 & 0.1071 & -0.0481 \\
\hline$\left(\left(0.7 / \mathfrak{g}_{5}\right), \mathfrak{e}_{3}, 0\right)$ & 0.0427 & 0.0349 & 0.3936 & 0.0976 & 0.0133 & -0.0045 & -0.0505 & 0.3107 \\
\hline$C_{j}=\sum_{i} w_{i} X_{i j}$ & 0.8271 & 0.9480 & 0.0026 & 0.2382 & 1.4126 & 0.1542 & 0.6377 & 1.4874 \\
\hline
\end{tabular}

TABle 8: Final scores.

\begin{tabular}{lcc}
\hline$L_{j}$ & $C_{j}$ & $\mathfrak{K}_{j}=L_{j}-C_{j}$ \\
\hline$L_{1}=-0.7936$ & $C_{1}=0.8271$ & $\mathfrak{K}_{1}=-1.6206$ \\
$L_{2}=0.6780$ & $C_{2}=0.9480$ & $\mathfrak{K}_{2}=-0.2700$ \\
$L_{3}=1.0982$ & $C_{3}=0.0026$ & $\mathfrak{K}_{3}=1.0957$ \\
$L_{4}=0.5857$ & $C_{4}=0.2382$ & $\mathfrak{K}_{4}=0.3475$ \\
$L_{5}=0.3210$ & $C_{5}=1.4126$ & $\mathfrak{K}_{5}=-1.0917$ \\
$L_{6}=0.8989$ & $C_{6}=0.1542$ & $\mathfrak{K}_{6}=0.7447$ \\
$L_{7}=0.8814$ & $C_{7}=0.6377$ & $\mathfrak{K}_{7}=0.2438$ \\
$L_{8}=-0.1797$ & $C_{8}=1.4874$ & $\mathfrak{K}_{8}=-1.6671$ \\
\hline
\end{tabular}

TABLE 9: Comparison of final scores by applying proposed model on the application in [29].

\begin{tabular}{lccccc}
\hline Hybrid models & $u_{1}$ & $u_{2}$ & $u_{3}$ & Ranking & Best option \\
\hline FPIFSESs [29] & 0.989 & -0.937 & 4.039 & $u_{3}>u_{1}>u_{2}$ & $u_{3}$ \\
FPPFSESs & 0.844 & -0.904 & 4.106 & $u_{3}>u_{1}>u_{2}$ & $u_{3}$ \\
FPFFSESs & 0.499 & -0.633 & 4.089 & $u_{3}>u_{1}>u_{2}$ \\
Proposed FP $q$ ROFSESs $(q=4)$ & 0.184 & -0.401 & 4.025 & $u_{3}>u_{1}>u_{2}$ \\
Proposed FPq ROFSESs $(q=5)$ & -0.082 & -0.224 & 3.927 & $u_{3}>u_{1}>u_{2}$ & $u_{3}$ \\
\hline
\end{tabular}




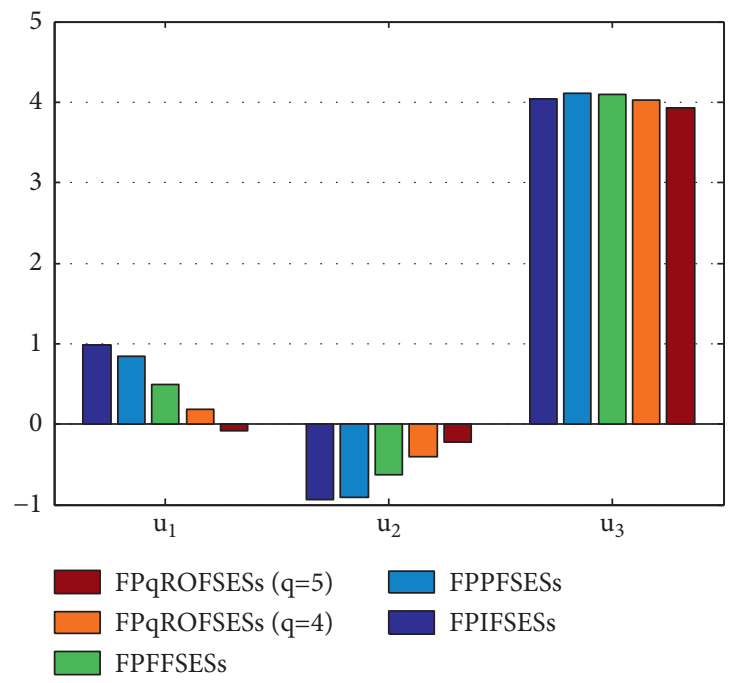

FIGURE 3: Comparison of final scores by applying the proposed model on the application in [29].

$\left\langle\left(\frac{0.3}{\mathfrak{S}_{5}}, \mathfrak{e}_{1}, 1\right)\left\{\frac{\mathfrak{y}_{1}}{(0.51,0.13)}, \frac{\mathfrak{y}_{2}}{(0.89,0.37)}, \frac{\mathfrak{y}_{3}}{(0.35,0.26)}, \frac{\mathfrak{y}_{4}}{(0.74,0.83)}, \frac{\mathfrak{y}_{5}}{(0.39,0.29)}, \frac{\mathfrak{y}_{6}}{(0.55,0.77)}, \frac{\mathfrak{y}_{7}}{(0.42,0.34)}, \frac{\mathfrak{y}_{8}}{(0.35,0.32)}\right\}\right\rangle$ $\left\langle\left(\frac{0.6}{\mathfrak{S}_{1}}, \mathfrak{e}_{2}, 1\right)\left\{\frac{\mathfrak{y}_{1}}{(0.50,0.70)}, \frac{\mathfrak{y}_{2}}{(0.39,0.24)}, \frac{\mathfrak{y}_{3}}{(0.29,0.37)}, \frac{\mathfrak{y}_{4}}{(0.511,0.75)}, \frac{\mathfrak{y}_{5}}{(0.27,0.43)}, \frac{\mathfrak{y}_{6}}{(0.34,0.13)}, \frac{\mathfrak{y}_{7}}{(0.80,0.60)}, \frac{\mathfrak{y}_{8}}{(0.47,0.62)}\right\}\right\rangle$, $\left\langle\left(\frac{0.2}{\mathfrak{g}_{2}}, \mathfrak{e}_{2}, 1\right)\left\{\frac{\mathfrak{y}_{1}}{(0.42,0.38)}, \frac{\mathfrak{y}_{2}}{(0.19,0.28)}, \frac{\mathfrak{y}_{3}}{(0.89,0.60)}, \frac{\mathfrak{y}_{4}}{(0.65,0.45)}, \frac{\mathfrak{y}_{5}}{(0.27,0.21)}, \frac{\mathfrak{y}_{6}}{(0.81,0.44)}, \frac{\mathfrak{y}_{7}}{(0.34,0.32)}, \frac{\mathfrak{y}_{8}}{(0.47,0.24)}\right\}\right\rangle$, $\left\langle\left(\frac{0.9}{\mathfrak{S}_{3}}, \mathfrak{e}_{2}, 1\right)\left\{\frac{\mathfrak{y}_{1}}{(0.61,0.11)}, \frac{\mathfrak{y}_{2}}{(0.43,0.16)}, \frac{\mathfrak{y}_{3}}{(0.83,0.27)}, \frac{\mathfrak{y}_{4}}{(0.78,0.12)}, \frac{\mathfrak{y}_{5}}{(0.25,0.27)}, \frac{\mathfrak{y}_{6}}{(0.29,0.23)}, \frac{\mathfrak{y}_{7}}{(0.88,0.72)}, \frac{\mathfrak{y}_{8}}{(0.11,0.27)}\right\}\right\rangle$, $\left\langle\left(\frac{0.7}{\mathfrak{S}_{4}}, \mathfrak{e}_{2}, 1\right)\left\{\frac{\mathfrak{y}_{1}}{(0.40,0.90)}, \frac{\mathfrak{y}_{2}}{(0.58,0.47)}, \frac{\mathfrak{y}_{3}}{(0.69,0.36)}, \frac{\mathfrak{y}_{4}}{(0.39,0.75)}, \frac{\mathfrak{y}_{5}}{(0.90,0.43)}, \frac{\mathfrak{y}_{6}}{(0.59,0.15)}, \frac{\mathfrak{y}_{7}}{(0.74,0.78)}, \frac{\mathfrak{y}_{8}}{(0.46,0.16)}\right\}\right\rangle$, $\left\langle\left(\frac{0.3}{\mathfrak{S}_{5}}, \mathfrak{e}_{2}, 1\right)\left\{\frac{\mathfrak{y}_{1}}{(0.64,0.20)}, \frac{\mathfrak{y}_{2}}{(0.37,0.56)}, \frac{\mathfrak{y}_{3}}{(0.81,0.52)}, \frac{\mathfrak{y}_{4}}{(0.56,0.46)}, \frac{\mathfrak{y}_{5}}{(0.22,0.65)}, \frac{\mathfrak{y}_{6}}{(0.94,0.34)}, \frac{\mathfrak{y}_{7}}{(0.32,0.34)}, \frac{\mathfrak{y}_{8}}{(0.21,0.27)}\right\}\right\rangle$, $\left\langle\left(\frac{0.6}{\mathfrak{g}_{1}}, \mathfrak{e}_{3}, 1\right)\left\{\frac{\mathfrak{y}_{1}}{(0.54,0.65)}, \frac{\mathfrak{y}_{2}}{(0.43,0.51)}, \frac{\mathfrak{y}_{3}}{(0.72,0.44)}, \frac{\mathfrak{y}_{4}}{(0.65,0.64)}, \frac{\mathfrak{y}_{5}}{(0.50,0.40)}, \frac{\mathfrak{y}_{6}}{(0.79,0.36)}, \frac{\mathfrak{y}_{7}}{(0.36,0.44)}, \frac{\mathfrak{y}_{8}}{(0.66,0.36)}\right\}\right\rangle$, $\left\langle\left(\frac{0.2}{\mathfrak{S}_{2}}, \mathfrak{e}_{3}, 1\right)\left\{\frac{\mathfrak{y}_{1}}{(0.50,0.15)}, \frac{\mathfrak{y}_{2}}{(0.80,0.50)}, \frac{\mathfrak{y}_{3}}{(0.90,0.60)}, \frac{\mathfrak{y}_{4}}{(0.70,0.20)}, \frac{\mathfrak{y}_{5}}{(0.80,0.10)}, \frac{\mathfrak{y}_{6}}{(0.30,0.20)}, \frac{\mathfrak{y}_{7}}{(0.50,0.20)}, \frac{\mathfrak{y}_{8}}{(0.40,0.70)}\right\}\right\rangle$, $\left\langle\left(\frac{0.9}{\mathfrak{S}_{3}}, \mathfrak{e}_{3}, 1\right)\left\{\frac{\mathfrak{y}_{1}}{(0.20,0.11)}, \frac{\mathfrak{y}_{2}}{(0.71,0.12)}, \frac{\mathfrak{y}_{3}}{(0.10,0.41)}, \frac{\mathfrak{y}_{4}}{(0.81,0.21)}, \frac{\mathfrak{y}_{5}}{(0.71,0.32)}, \frac{\mathfrak{y}_{6}}{(0.81,0.61)}, \frac{\mathfrak{y}_{7}}{(0.80,0.16)}, \frac{\mathfrak{y}_{8}}{(0.40,0.14)}\right\}\right\rangle$, $\left\langle\left(\frac{0.7}{\mathfrak{S}_{4}}, \mathfrak{e}_{3}, 1\right)\left\{\frac{\mathfrak{y}_{1}}{(0.44,0.72)}, \frac{\mathfrak{y}_{2}}{(0.87,0.45)}, \frac{\mathfrak{y}_{3}}{(0.62,0.42)}, \frac{\mathfrak{y}_{4}}{(0.52,0.62)}, \frac{\mathfrak{y}_{5}}{(0.77,0.63)}, \frac{\mathfrak{y}_{6}}{(0.65,0.56)}, \frac{\mathfrak{y}_{7}}{(0.80,0.30)}, \frac{\mathfrak{y}_{8}}{(0.45,0.25)}\right\}\right\rangle$, $\left\langle\left(\frac{0.3}{\mathfrak{S}_{5}}, \mathfrak{e}_{3}, 1\right)\left\{\frac{\mathfrak{y}_{1}}{(0.27,0.31)}, \frac{\mathfrak{y}_{2}}{(0.36,0.25)}, \frac{\mathfrak{y}_{3}}{(0.63,0.36)}, \frac{\mathfrak{y}_{4}}{(0.71,0.81)}, \frac{\mathfrak{y}_{5}}{(0.61,0.53)}, \frac{\mathfrak{y}_{6}}{(0.51,0.42)}, \frac{\mathfrak{y}_{7}}{(0.56,0.45)}, \frac{\mathfrak{y}_{8}}{(0.52,0.48)}\right\}\right\rangle$, $\left\langle\left(\frac{0.6}{\mathfrak{g}_{1}}, \mathfrak{e}_{1}, 0\right)\left\{\frac{\mathfrak{y}_{1}}{(0.30,0.14)}, \frac{\mathfrak{y}_{2}}{(0.90,0.16)}, \frac{\mathfrak{y}_{3}}{(0.80,0.70)}, \frac{\mathfrak{y}_{4}}{(0.70,0.40)}, \frac{\mathfrak{y}_{5}}{(0.80,0.30)}, \frac{\mathfrak{y}_{6}}{(0.50,0.11)}, \frac{\mathfrak{y}_{7}}{(0.61,0.12)}, \frac{\mathfrak{y}_{8}}{(0.60,0.30)}\right\}\right\rangle$, $\left\langle\left(\frac{0.2}{\mathfrak{g}_{2}}, \mathfrak{e}_{1}, 0\right)\left\{\frac{\mathfrak{y}_{1}}{(0.89,0.43)}, \frac{\mathfrak{y}_{2}}{(0.69,0.33)}, \frac{\mathfrak{y}_{3}}{(0.54,0.93)}, \frac{\mathfrak{y}_{4}}{(0.28,0.57)}, \frac{\mathfrak{y}_{5}}{(0.75,0.11)}, \frac{\mathfrak{y}_{6}}{(0.70,0.11)}, \frac{\mathfrak{y}_{7}}{(0.81,0.74)}, \frac{\mathfrak{y}_{8}}{(0.94,0.19)}\right\}\right\rangle$, $\left\langle\left(\frac{0.9}{\mathfrak{S}_{3}}, \mathfrak{e}_{1}, 0\right)\left\{\frac{\mathfrak{y}_{1}}{(0.80,0.50)}, \frac{\mathfrak{y}_{2}}{(0.20,0.19)}, \frac{\mathfrak{y}_{3}}{(0.23,0.20)}, \frac{\mathfrak{y}_{4}}{(0.50,0.35)}, \frac{\mathfrak{y}_{5}}{(0.43,0.20)}, \frac{\mathfrak{y}_{6}}{(0.54,0.70)}, \frac{\mathfrak{y}_{7}}{(0.80,0.68)}, \frac{\mathfrak{y}_{8}}{(0.79,0.50)}\right\}\right\rangle$, 


$$
\begin{aligned}
& \left\langle\left(\frac{0.7}{\mathfrak{g}_{4}}, \mathfrak{e}_{1}, 0\right)\left\{\frac{\mathfrak{y}_{1}}{(0.22,0.15)}, \frac{\mathfrak{y}_{2}}{(0.24,0.11)}, \frac{\mathfrak{y}_{3}}{(0.29,0.11)}, \frac{\mathfrak{y}_{4}}{(0.22,0.10)}, \frac{\mathfrak{y}_{5}}{(0.39,0.37)}, \frac{\mathfrak{y}_{6}}{(0.45,0.35)}, \frac{\mathfrak{y}_{7}}{(0.38,0.27)}, \frac{\mathfrak{y}_{8}}{(0.54,0.22)}\right\}\right\rangle, \\
& \left\langle\left(\frac{0.3}{\mathfrak{g}_{5}}, \mathfrak{e}_{1}, 0\right)\left\{\frac{\mathfrak{y}_{1}}{(0.70,0.40)}, \frac{\mathfrak{y}_{2}}{(0.60,0.50)}, \frac{\mathfrak{y}_{3}}{(0.25,0.60)}, \frac{\mathfrak{y}_{4}}{(0.60,0.37)}, \frac{\mathfrak{y}_{5}}{(0.70,0.44)}, \frac{\mathfrak{y}_{6}}{(0.57,0.90)}, \frac{\mathfrak{y}_{7}}{(0.72,0.40)}, \frac{\mathfrak{y}_{8}}{(0.81,0.20)}\right\}\right\rangle, \\
& \left\langle\left(\frac{0.6}{\mathfrak{g}_{1}}, \mathfrak{e}_{2}, 0\right)\left\{\frac{\mathfrak{y}_{1}}{(0.25,0.17)}, \frac{\mathfrak{y}_{2}}{(0.31,0.13)}, \frac{\mathfrak{y}_{3}}{(0.42,0.31)}, \frac{\mathfrak{y}_{4}}{(0.46,0.34)}, \frac{\mathfrak{y}_{5}}{(0.70,0.18)}, \frac{\mathfrak{y}_{6}}{(0.40,0.34)}, \frac{\mathfrak{y}_{7}}{(0.20,0.15)}, \frac{\mathfrak{y}_{8}}{(0.31,0.13)}\right\}\right\rangle, \\
& \left\langle\left(\frac{0.2}{\mathfrak{g}_{2}}, \mathfrak{e}_{2}, 0\right)\left\{\frac{\mathfrak{y}_{1}}{(0.90,0.30)}, \frac{\mathfrak{y}_{2}}{(0.30,0.12)}, \frac{\mathfrak{y}_{3}}{(0.31,0.12)}, \frac{\mathfrak{y}_{4}}{(0.70,0.38)}, \frac{\mathfrak{y}_{5}}{(0.48,0.31)}, \frac{\mathfrak{y}_{6}}{(0.61,0.20)}, \frac{\mathfrak{y}_{7}}{(0.75,0.60)}, \frac{\mathfrak{y}_{8}}{(0.84,0.50)}\right\}\right\rangle, \\
& \left\langle\left(\frac{0.9}{\mathfrak{g}_{3}}, \mathfrak{e}_{2}, 0\right)\left\{\frac{\mathfrak{y}_{1}}{(0.27,0.19)}, \frac{\mathfrak{y}_{2}}{(0.26,0.14)}, \frac{\mathfrak{y}_{3}}{(0.24,0.12)}, \frac{\mathfrak{y}_{4}}{(0.24,0.12)}, \frac{\mathfrak{y}_{5}}{(0.90,0.21)}, \frac{\mathfrak{y}_{6}}{(0.13,0.25)}, \frac{\mathfrak{y}_{7}}{(0.39,0.29)}, \frac{\mathfrak{y}_{8}}{(0.51,0.38)}\right\}\right\rangle, \\
& \left\langle\left(\frac{0.7}{\mathfrak{g}_{4}}, \mathfrak{e}_{2}, 0\right)\left\{\frac{\mathfrak{y}_{1}}{(0.50,0.20)}, \frac{\mathfrak{y}_{2}}{(0.22,0.10)}, \frac{\mathfrak{y}_{3}}{(0.23,0.22)}, \frac{\mathfrak{y}_{4}}{(0.45,0.11)}, \frac{\mathfrak{y}_{5}}{(0.51,0.40)}, \frac{\mathfrak{y}_{6}}{(0.81,0.64)}, \frac{\mathfrak{y}_{7}}{(0.82,0.73)}, \frac{\mathfrak{y}_{8}}{(0.86,0.41)}\right\}\right\rangle, \\
& \left\langle\left(\frac{0.3}{\mathfrak{g}_{5}}, \mathfrak{e}_{2}, 0\right)\left\{\frac{\mathfrak{y}_{1}}{(0.21,0.17)}, \frac{\mathfrak{y}_{2}}{(0.25,0.16)}, \frac{\mathfrak{y}_{3}}{(0.11,0.22)}, \frac{\mathfrak{y}_{4}}{(0.21,0.70)}, \frac{\mathfrak{y}_{5}}{(0.45,0.38)}, \frac{\mathfrak{y}_{6}}{(0.45,0.31)}, \frac{\mathfrak{y}_{7}}{(0.17,0.12)}, \frac{\mathfrak{y}_{8}}{(0.81,0.27)}\right\}\right\rangle, \\
& \left\langle\left(\frac{0.6}{\mathfrak{I}_{1}}, \mathfrak{e}_{3}, 0\right)\left\{\frac{\mathfrak{y}_{1}}{(0.62,0.11)}, \frac{\mathfrak{y}_{2}}{(0.43,0.20)}, \frac{\mathfrak{y}_{3}}{(0.45,0.11)}, \frac{\mathfrak{y}_{4}}{(0.64,0.19)}, \frac{\mathfrak{y}_{5}}{(0.69,0.21)}, \frac{\mathfrak{y}_{6}}{(0.71,0.17)}, \frac{\mathfrak{y}_{7}}{(0.63,0.15)}, \frac{\mathfrak{y}_{8}}{(0.63,0.45)}\right\}\right\rangle, \\
& \left\langle\left(\frac{0.2}{\mathfrak{g}_{2}}, \mathfrak{e}_{3}, 0\right)\left\{\frac{\mathfrak{y}_{1}}{(0.61,0.12)}, \frac{\mathfrak{y}_{2}}{(0.45,0.16)}, \frac{\mathfrak{y}_{3}}{(0.31,0.13)}, \frac{\mathfrak{y}_{4}}{(0.55,0.15)}, \frac{\mathfrak{y}_{5}}{(0.34,0.10)}, \frac{\mathfrak{y}_{6}}{(0.30,0.12)}, \frac{\mathfrak{y}_{7}}{(0.39,0.17)}, \frac{\mathfrak{y}_{8}}{(0.62,0.17)}\right\}\right\rangle, \\
& \left\langle\left(\frac{0.9}{\mathfrak{g}_{3}}, \mathfrak{e}_{3}, 0\right)\left\{\frac{\mathfrak{y}_{1}}{(0.72,0.32)}, \frac{\mathfrak{y}_{2}}{(0.73,0.25)}, \frac{\mathfrak{y}_{3}}{(0.70,0.50)}, \frac{\mathfrak{y}_{4}}{(0.28,0.19)}, \frac{\mathfrak{y}_{5}}{(0.83,0.20)}, \frac{\mathfrak{y}_{6}}{(0.56,0.32)}, \frac{\mathfrak{y}_{7}}{(0.59,0.15)}, \frac{\mathfrak{y}_{8}}{(0.78,0.34)}\right\}\right\rangle, \\
& \left\langle\left(\frac{0.7}{\mathfrak{g}_{4}}, \mathfrak{e}_{3}, 0\right)\left\{\frac{\mathfrak{y}_{1}}{(0.39,0.24)}, \frac{\mathfrak{y}_{2}}{(0.89,0.60)}, \frac{\mathfrak{y}_{3}}{(0.12,0.78)}, \frac{\mathfrak{y}_{4}}{(0.27,0.25)}, \frac{\mathfrak{y}_{5}}{(0.29,0.23)}, \frac{\mathfrak{y}_{6}}{(0.46,0.16)}, \frac{\mathfrak{y}_{7}}{(0.64,0.20)}, \frac{\mathfrak{y}_{8}}{(0.37,0.56)}\right\}\right\rangle, \\
& \left.\left\langle\left(\frac{0.3}{\mathfrak{g}_{5}}, \mathfrak{e}_{3}, 0\right)\left\{\frac{\mathfrak{y}_{1}}{(0.58,0.47)}, \frac{\mathfrak{y}_{2}}{(0.53,0.37)}, \frac{\mathfrak{y}_{3}}{(0.83,0.20)}, \frac{\mathfrak{y}_{4}}{(0.65,0.45)}, \frac{\mathfrak{y}_{5}}{(0.43,0.27)}, \frac{\mathfrak{y}_{6}}{(0.13,0.34)}, \frac{\mathfrak{y}_{7}}{(0.34,0.56)}, \frac{\mathfrak{y}_{8}}{(0.81,0.52)}\right\}\right\rangle\right\} .
\end{aligned}
$$

The committee uses Algorithm 1 for the selection of the "Best News Channel."

Table 5 represents the score table for the $\mathrm{FP}^{5}$ ROFSES defined above. Tables 6 and 7 represent the score tables for agree- and disagree-FP ${ }^{5}$ ROFSESs, respectively, along with the agree and disagree accumulated scores.

Using agree and disagree accumulated scores in Tables 6 and 7, Table 8 provides the final scores. From the final scores table, it can be seen that $\boldsymbol{K}_{m}=\max \left(\boldsymbol{K}_{j}\right)=\boldsymbol{K}_{3}$; hence, the news channel $\mathfrak{y}_{3}$ is awarded as the "Best News Channel" by the committee and the organization.

\section{Comparison}

These days, experts believe that fuzzy parameterized extensions of the soft set model and its hybrid structures with other uncertainty theories are playing a crucial role in solving several daily-life decision-making problems. Till date, the mathematical tools considering above-mentioned topic in hand are FPFSSs [20], IFPFSSs [22], IFPIFSSs [24], IVIFP-IVIFSSs [25], FPFSESs [45], and FPIFSESs [29]. Inspection of researches completed in the last few decades proves the significance of uncertain hybrid models toward this topic. Clearly, our proposed hybrid model, $\mathrm{FP}^{q}$ ROFSESs, generalized the FPIFSESs [29]. Note that FPIFSESs
[29], fuzzy parameterized Pythagorean FSESs (FPPFSESs), and fuzzy parameterized Fermatean FSESs (FPFFSESs) are particular cases of our developed $\mathrm{FP}^{q}$ ROFSES model for $q=1,2$ and $q=3$, respectively. One cannot apply the existing FPIFSES model to the proposed applications in Section 3. Thus, to clearly observe the advantages of the developed model and its comparative analysis with existing models, it is applied to the application in [29] and obtains similar results for different values of parameter " $q$," which are computed in Table 9 and displayed in Figure 3. In [29], the authors have not used weights in the group decisionmaking process, which was an essential part of their new construction. So, there is a flaw in their model. In our proposed method, we not only cover this issue (that is, we have utilized these weights in the developed group decisionmaking method, see Algorithm 1; Steps 5 and 6) but also provided its generalization. Thus, our proposed hybrid model applicability scope is wider than existing models, including FPIFSESs [29] and FPFSES [45].

4.1. Advantages of the Initiated Model. From the inspection of the recent decade, one can easily observe various developments in the hybrid models containing fuzzy parameterized soft sets as one of their components. All these 
models are inefficient to tackle data in a $q$-ROF environment with $n$ experts where $n \geq 1$. A generalized hybrid approach is currently required, which maintains the features of more than one existing model. With the motivation of these concerns, a new hybrid model called $\mathrm{FP}^{q}$ ROFSES is initiated. In our developed model, the estimations of all experts are examined in a $q$-ROF environment. The developed model's applicability scope is wider than various existing models, including FPFSSs [20], FPFSESs [45], and FPIFSESs [29] because it is an efficient extension of all these models. It can be easily seen that the existing MAGDM method, namely, FPIFSESs, cannot address the MADM situations as studied in Section 3. Thus, the developed model is very reliable and flexible for dealing with imprecise fuzzy parameterized $q$-ROF soft expert information, specifically, if the available information is collected from multiple experts in a $q$-ROF environment.

\section{Conclusions}

The theory of $q$-ROFSs has proved to be a strong tool for dealing with high levels of uncertainties in many practical situations than IFSs, PFSs, and FFSs; thus, it is a basic component of many mathematical hybrid decision-making models for dealing with such complex decisive situations. The fuzzy parameterized soft sets and their extensions are more efficient in dealing with scenarios considering much preference of some of the parameters over the others, thus getting more precisely to the required decisions. Similarly, for MAGDM situations, SESs as an efficient model provide the facility of considering multiple experts' opinions in one place. This article extends the hybrid model FPIFSESs to a more generalized novel hybrid structure called $\mathrm{FP}^{q}$ ROFSESs, which is actually a combination of $q$-ROFSs [5] and FPSESs [46]. When $q=1$, the proposed model reduces to the FPIFSES model [29], and when $q=2$ and $q=3$, it degenerates into FPPFSESs and FPFFSESs, respectively. Some essential basic notions including subset, complement, AND operation, OR operation, intersection, and union are studied coupled with illustrative examples. De Morgan's laws under $\mathrm{FP}^{q}$ ROFSESs are verified. Moreover, to show the applicability and efficiency of the proposed model, two reallife applications, including the selection of the best site for cafe outlet and Best News Channel, are provided and solved under $\mathrm{FP}^{q}$ ROFSESs with the help of a developed algorithm. Finally, a comparison of the developed group decisionmaking method under $\mathrm{FP}^{q}$ ROFSESs is studied with few existing approaches, including FPIFSESs [29]. This comparative analysis in Section 4 shows that the developed model's applicability and reliability scope is higher than existing models, including $q$-ROFSSs and FPIFSESs. During the construction of our initiated model, we observed that it has two major limitations. Firstly, the proposed model fails in a situation if weights are given in the form of an intuitionistic fuzzy environment. Secondly, it fails if experts provide their estimations in the form of an interval-valued $q$-ROF environment. In the future, to remove these drawbacks of our model, we are planning to expand our work with the following models: (a) intuitionistic fuzzy parameterized $q$-rung orthopair fuzzy soft expert sets using the idea in [24], (b) fuzzy parameterized interval-valued q-rung orthopair fuzzy soft expert sets using the idea in [25], (c) fuzzy parameterized complex interval-valued $q$-rung orthopair fuzzy soft expert sets with aggregation operators using the concepts in [7], and (d) fuzzy parameterized complex $q$-rung orthopair fuzzy soft expert sets using [8].

\section{Data Availability}

No supporting data are required for the results of the paper. All necessary details are available within the article.

\section{Ethical Approval}

This article does not contain any studies with human participants or animals performed by any of the authors.

\section{Conflicts of Interest}

The authors declare that they have no conflicts of interest regarding the publication of this article.

\section{References}

[1] L. A. Zadeh, "Fuzzy sets," Information and Control, vol. 8, no. 3, pp. 338-353, 1965.

[2] K. T. Atanassov, "Intuitionistic fuzzy sets," Fuzzy Sets and Systems, vol. 20, no. 1, pp. 87-96, 1986.

[3] R. R. Yager, "Pythagorean fuzzy subsets," in Proceedings of the 2013 Joint IFSA World Congress and NAFIPS Annual Meeting (IFSA/NAFIPS), 2013.

[4] T. Senapati and R. R. Yager, "Fermatean fuzzy sets," Journal of Ambient Intelligence and Humanized Computing, vol. 11, no. 2, pp. 663-674, 2020.

[5] R. R. Yager, "Generalized orthopair fuzzy sets," IEEE Transactions on Fuzzy Systems, vol. 25, no. 5, pp. 1222-1230, 2016.

[6] T. Shaheen, M. I. Ali, and H. Toor, "Why do we need q-rung orthopair fuzzy sets? Some evidence established via mass assignment," International Journal of Intelligent Systems, vol. 36, no. 10, pp. 5493-5505, 2021.

[7] H. Garg, Z. Ali, and T. Mahmood, "Algorithms for complex interval-valued $q$-rung orthopair fuzzy sets in decision making based on aggregation operators, AHP, and TOPSIS," Expert Systems, vol. 38, no. 1, 2020.

[8] H. Garg, "New exponential operation laws and operators for interval-valued $q$-rung orthopair fuzzy sets in group decision making process," Neural Computing \& Applications, pp. 1-27, 2021.

[9] P. Liu and P. Wang, "Some $q$-rung orthopair fuzzy aggregation operators and their applications to multiple-attribute decision making," International Journal of Intelligent Systems, vol. 33, no. 2, pp. 259-280, 2018.

[10] X. Peng, H. Huang, and Z. Luo, " $q$-rung orthopair fuzzy decision-making framework for integrating mobile edge caching scheme preferences," International Journal of Intelligent Systems, vol. 36, no. 5, pp. 2229-2266, 2021.

[11] X. Peng, J. Dai, and H. Garg, "Exponential operation and aggregation operator for $q$-rung orthopair fuzzy set and their decision-making method with a new score function," International Journal of Intelligent Systems, vol. 33, no. 11, pp. 2255-2282, 2018. 
[12] D. Molodtsov, "Soft set theory-first results," Computers and Mathematics with Applications, vol. 37, no. 2, pp. 19-31, 1999.

[13] P. K. Maji, A. R. Roy, and R. Biswas, "Soft set theory," Computers and Mathematics with Applications, vol. 54, no. 45, pp. 555-562, 2003.

[14] P. K. Maji, A. R. Roy, and R. Biswas, "Fuzzy soft sets," Journal of Fuzzy Mathematics, vol. 9, no. 3, pp. 589-602, 2001.

[15] P. K. Maji, R. Biswas, and A. R. Roy, "Intuitionistic fuzzy soft sets," Journal of Fuzzy Mathematics, vol. 9, no. 3, pp. 677-692, 2001.

[16] M. T. Hamid, M. Riaz, and D. Afzal, "Novel MCGDM with qrung orthopair fuzzy soft sets and TOPSIS approach under $q$ rung orthopair fuzzy soft topology," Journal of Intelligent and Fuzzy Systems, vol. 39, no. 3, pp. 3853-3871, 2020.

[17] G. Ali, H. Alolaiyan, D. Pamucar, M. Asif, and N. Lateef, "A novel MADM framework under q-rung orthopair fuzzy bipolar soft sets," Mathematics, vol. 9, p. 2163, 2021.

[18] N. Alkan and C. Kahraman, "Evaluation of government strategies against COVID-19 pandemic using q-rung orthopair fuzzy TOPSIS method," Applied Soft Computing, vol. 110, Article ID 107653, 2021.

[19] N. Aman and S. Enginoglu, "FP-soft set theory and its applications," Annals of Fuzzy Mathematics and Informatics, vol. 2, no. 2, pp. 219-226, 2011.

[20] N. aman, F. Çıtak, and S. Enginoglu, "Fuzzy parameterized fuzzy soft set theory and its applications," Turkish Journal of Fuzzy System, vol. 1, no. 1, pp. 21-35, 2010.

[21] I. Deli and N. Çağman, "Intuitionistic fuzzy parameterized soft set theory and its decision making," Applied Soft Computing, vol. 28, pp. 109-113, 2015.

[22] E. E. Yagubi and A. R. Salleh, "Intuitionistic fuzzy parameterised fuzzy soft set," Journal of Quality Measurement and Analysis, vol. 9, no. 2, pp. 73-81, 2013.

[23] S. Enginolu and B. Arslan, "Intuitionistic fuzzy parameterized intuitionistic fuzzy soft matrices and their application in decision-making," Computational and Applied Mathematics, vol. 39, no. 4 , pp. 1-20, 2020.

[24] F. Karaaslan, "Intuitionistic fuzzy parameterized intuitionistic fuzzy soft sets with applications in decision making," Annals of Fuzzy Mathematics and Informatics, vol. 11, no. 4, pp. 607-619, 2016.

[25] T. Aydın and S. Enginolu, "Interval-valued intuitionistic fuzzy parameterized interval-valued intuitionistic fuzzy soft sets and their application in decision-making," Journal of Ambient Intelligence and Humanized Computing, vol. 12, no. 1, pp. 1541-1558, 2021.

[26] S. Alkhazaleh and A. R. Salleh, "Soft expert sets," Advances in Decision Sciences, vol. 2011, Article ID 757868, 12 pages, 2011.

[27] S. Alkhazaleh and A. R. Salleh, "Fuzzy soft expert set and its application," Applied Mathematics, vol. 5, no. 9, pp. 1349-1368, 2014.

[28] G. Ali and M. Akram, "Decision-making method based on fuzzy $N$-soft expert sets," Arabian Journal for Science and Engineering, vol. 45, no. 12, pp. 10381-10400, 2020.

[29] G. Selvachandran and A. R. Salleh, "Fuzzy parameterized intuitionistic fuzzy soft expert set theory and its application in decision making," International Journal of Soft Computing, vol. 11, pp. 52-63, 2016.

[30] G. Ali, G. Muhiuddin, A. Adeel, and M. Zain Ul Abidin, "Ranking effectiveness of COVID-19 tests using fuzzy bipolar soft expert sets," Mathematical Problems in Engineering, vol. 2021, Article ID 5874216, 19 pages, 2021.
[31] M. Sarwar, M. Akram, and S. Shahzadi, "Bipolar fuzzy soft information applied to hypergraphs," Soft Computing, vol. 25, no. 5, pp. 3417-3439, 2021.

[32] M. Sarwar, M. Akram, and P. Liu, "An integrated rough ELECTRE II approach for risk evaluation and effects analysis in automatic manufacturing process," Artificial Intelligence Review, vol. 54, no. 6, pp. 4449-4481, 2021.

[33] M. Sarwar, "Decision-making approaches based on color spectrum and D-TOPSIS method under rough environment," Computational and Applied Mathematics, vol. 39, no. 4, pp. 1-32, 2020.

[34] M. Akram, G. Ali, M. A. Butt, and J. C. R. Alcantud, "Novel MCGDM analysis under m-polar fuzzy soft expert sets," Neural Computing \& Applications, vol. 33, no. 18, pp. 12051-12071, 2021.

[35] M. Akram, G. Ali, and J. C. R. Alcantud, "Parameter reduction analysis under interval-valued $m$-polar fuzzy soft information," Artificial Intelligence Review, vol. 54, pp. 55415582, 2021.

[36] G. Ali and M. N. Ansari, "Multiattribute decision-making under Fermatean fuzzy bipolar soft framework," Granular Computing, 2021.

[37] Z. Ali, T. Mahmood, T. Mahmood, K. Ullah, and Q. Khan, "Einstein geometric aggregation operators using a novel complex interval-valued Pythagorean fuzzy setting with application in green supplier chain management," Reports in Mechanical Engineering, vol. 2, no. 1, pp. 105-134, 2021.

[38] A. Alosta, O. Elmansuri, and I. Badi, "Resolving a location selection problem by means of an integrated AHP-RAFSI approach," Reports in Mechanical Engineering, vol. 2, no. 1, pp. 135-142, 2021.

[39] F. Feng, H. Fujita, M. I. Ali, R. R. Yager, and X. Liu, "Another view on generalized intuitionistic fuzzy soft sets and related multi attribute decision making methods," IEEE Transactions on Fuzzy Systems, vol. 27, pp. 474-488, 2018.

[40] H. Garg, A. Keikha, and H. Mishmast Nehi, "Multiple-attribute decision-making problem using TOPSIS and choquet integral with hesitant fuzzy number information," Mathematical Problems in Engineering, vol. 2020, Article ID 9874951, 12 pages, 2020.

[41] D. Pamucar, D. Macura, M. Tavana, D. Božanić, and N. Knežević, "An integrated rough group multicriteria decision-making model for the ex-ante prioritization of infrastructure projects: the Serbian railways case," SocioEconomic Planning Sciences, 2021, In press, Article ID 101098.

[42] D. Pamučar, "Multi-criteria model for the selection of construction materials: an approach based on fuzzy logic," Tehnički Vjesnik, vol. 27, no. 5, pp. 1531-1543, 2020.

[43] G. Petrović, J. Mihajlović, Ž. Ćojbašić, M. Madić, and D. Marinković, "Comparison of three fuzzy MCDM methods for solving the supplier selection problem," Facta Universitatis Series: Mechanical Engineering, vol. 17, no. 3, pp. 455-469, 2019.

[44] K. R. Ramakrishnan and S. Chakraborty, "A cloud TOPSIS model for green supplier selection," Facta Universitatis Series: Mechanical Engineering, vol. 18, no. 3, pp. 375-397, 2020.

[45] A. Hazaymeh, I. B. Abdullah, Z. Balkhi, and R. Ibrahim, "Fuzzy parameterized fuzzy soft expert set," Applied Mathematical Sciences, vol. 6, no. 112, pp. 5547-5564, 2012.

[46] M. Bashir and A. R. Salleh, "Fuzzy parameterized soft expert set," Abstract and Applied Analysis, vol. 2012, Article ID 258361, 15 pages, 2012. 